UDC 539.3

DOI: http://dx.doi.org/10.20535/2521-1943.2017.80.113757

\title{
Electricity generation in the world and Ukraine: Current status and future developments*
}

\author{
Alexander Zvorykin ${ }^{1} \bullet$ Igor Pioro $^{2} \bullet$ Nataliia Fialko $^{3}$ \\ 1 - Igor Sikorsky Kyiv Polytechnic Institute, Kyiv, Ukraine \\ 2 - University of Ontario Institute of Technology, Canada \\ 3 - Institute of Engineering Thermophysics of National Academy of Sciences of Ukraine
}

Received: 19 August 2017 / Accepted: 05 September 2017

\begin{abstract}
Electricity generation is the key factor for advances in industry, agriculture, technology and the level of living. Also, strong power industry with diverse energy sources is very important for country independence. In general, electricity can be generated from: 1) non-renewable energy sources such as coal, natural gas, oil, and nuclear; and 2) renewable energy sources such as hydro, biomass, wind, geothermal, solar, and wave power. However, the major energy sources for electricity generation in the world are: 1) thermal power - primarily using coal ( 40\%) and secondarily natural gas ( 23\%); 2) "large" hydro power plants $(\sim 17 \%)$ and 3$)$ nuclear power from various reactor designs ( 11\%). The rest of the energy sources for electricity generation is from using oil ( 4\%) and renewable sources such as biomass, wind, geothermal and solar ( 5\%), which have just visible impact in selected countries. In addition, energy sources, such as wind and solar, and some others, like tidal and wave-power, are intermittent from depending on Mother Nature. And cannot be used alone for industrial electricity generation.

Nuclear power in Ukraine is the most important source of electricity generation in the country. Currently, Ukrainian Nuclear Power Plants (NPPS) generate about $45.5 \%$ of the total electricity followed with coal generation - 38\%, gas generation $9.6 \%$ and the rest is based on renewable sources, mainly on hydro power plants - 5.9\%. Nuclear-power industry is based on four NPPs (15 Pressurized Water Reactors (PWRs) including the largest one in Europe - Zaporizhzhya NPP with about 6,000 MWel gross installed capacity. Two of these 15 reactors have been built and put into operation in 70-s, ten in 80-s, one in 90-s and just two in 2004. Therefore, based on an analysis of the world power reactors in terms of their maximum years of operation (currently, the oldest reactors are $\sim 45$-year old) several projections have been made for future of the nuclear-power industry in the world and Ukraine. Unfortunately, all these projections are quite pessimistic. There is a possibility that around 2030-2040 the vast majority of the world reactors and Ukrainian reactors will be shut down, and, in particular, Ukraine can be left without the basic and vital source of electricity generation.
\end{abstract}

Keywords: Electricity Generation, Nuclear Power Plant, Nuclear-Power Reactor, Thermal Efficiency, Capacity Factor.

\section{INTRODUCTION}

It is well known that electricity generation is the key factor for advances in industry, agriculture, technology and the level of living (for details, see Table 1 and Figure 1) (Handbook, 2016; Pioro and Duffey, 2015; Pioro and Kirillov, 2013a). Also, strong power industry with diverse energy sources is very important for country independence. In general, electricity can be generated from: 1) non-renewable energy sources such as coal, natural gas, oil, and nuclear; and 2) renewable energy sources such as hydro, biomass, wind, geothermal, solar, and wave power. However, as of today the major energy sources for electricity generation in the world (for details, see Figure 1a) are: 1) thermal power primarily using coal $(\sim 40 \%)$ and secondarily - natural gas $(\sim 23 \%)$; 2$)$ "large" hydro power plants $(\sim 17 \%)$ and 3$)$ nuclear power from various reactor designs $(\sim 11 \%)$. The rest of the energy sources for electricity generation is from using oil $(\sim 4 \%)$ and renewable sources such as biomass, wind, geothermal and solar $(\sim 5 \%)$, which have just visible impact in selected countries (for details, see Figures 1 and 2; Tables 2 and 3). In addition, energy sources, such as wind and solar, and some others, like tidal and wave-power, are intermittent from depending on Mother Nature (see Figures 3 and 4; for more details, see Handbook, 2016; and Pioro and Duffey, 2015), and cannot be used alone for industrial electricity generation.

* - editorial invited article [на замовлення редакції]

$\begin{array}{lr}\square & \text { Igor Pioro } \\ \text { Igor.Pioro@uoit.ca } & \square \text { Alexander Zvorykin } \\ & \text { panet12388@gmail.com }\end{array}$

(C) Mechanics and Advanced Technologies, 2017 
Table 1

Electrical-Energy Consumption (EEC) per capita in selected countries (listed here just for reference purposes) (based on Handbook, 2016; The World Fact Book, 2013; Human Development Report, 2013)

\begin{tabular}{|c|c|c|c|c|c|c|}
\hline \multirow[t]{2}{*}{ No } & \multirow[t]{2}{*}{ Country } & \multirow{2}{*}{$\begin{array}{c}\text { Population } \\
\text { Millions }\end{array}$} & \multicolumn{2}{|c|}{ Electrical Energy Consumption } & \multicolumn{2}{|c|}{ HDI* ( 2014) } \\
\hline & & & TW h/year & W/Capita & Rank & Value \\
\hline 1 & Norway & 5.2 & 120.5 & 2618 & 1 & 0.944 \\
\hline 2 & Australia & 22.8 & 222.6 & 1116 & 2 & 0.935 \\
\hline 3 & Germany & 80.9 & 540.1 & 762 & 6 & 0.916 \\
\hline 4 & USA & 321.4 & 3832.0 & 1360 & 8 & 0.915 \\
\hline 5 & Canada & 35.1 & 524.8 & 1706 & 9 & 0.913 \\
\hline 6 & UK & 64.1 & 319.1 & 568 & 14 & 0.907 \\
\hline 7 & Japan & 126.9 & 921.0 & 828 & 20 & 0.891 \\
\hline 8 & France & 66.6 & 451.1 & 773 & 22 & 0.888 \\
\hline 9 & Italy & 61.9 & 303.1 & 559 & 27 & 0.873 \\
\hline 10 & Russia & 142.4 & 1037.0 & 831 & 50 & 0.798 \\
\hline 11 & Brazil & 204.3 & 483.5 & 270 & 75 & 0.755 \\
\hline 12 & Ukraine & 44.4 & 159.8 & 410 & 81 & 0.747 \\
\hline 13 & China & $1,367.5$ & 5523.0 & 461 & 90 & 0.727 \\
\hline 14 & World (average) & $7,256.5$ & $19,710.0$ & 310 & 103 & 0.711 \\
\hline 15 & India & $1,251.7$ & 864.7 & 79 & 130 & 0.609 \\
\hline 16 & Afghanistan & 32.6 & 3.9 & 14 & 171 & 0.465 \\
\hline 17 & Chad & 11.6 & 0.2 & 2 & 185 & 0.392 \\
\hline 18 & Niger & 18.1 & 0.9 & 6 & 188 & 0.348 \\
\hline
\end{tabular}

* EEC, $\frac{\mathrm{W}}{\text { Capita }}=\frac{\mathrm{EEC}, \frac{\mathrm{TW} \mathrm{h}}{\mathrm{year}} \times \frac{10^{12}}{365 \text { days } \times 24 \mathrm{~h}}}{\text { Population, Millions } \times 10^{6}}$.

** HDI - Human Development Index by United Nations (UN); HDI is a comparative measure of life expectancy, literacy, education and standards of living for countries worldwide. HDI is calculated by the following formula: HDI $=\sqrt[3]{\mathrm{LEI} \times \mathrm{EI} \times \mathrm{II}}$, where LEI $-\underline{\mathrm{Life}}$ Expectancy Index, EI - Education Index, and II - Income Index. It is used to distinguish whether the country is a developed, a developing or an under-developed country, and also to measure the impact of economic policies on quality of life. Countries fall into four broad human-development categories, each of which comprises 42 countries: 1) Very high - 42 countries; 2) high - 43; 3 ) medium - 42; and 4) low - 42 (Wikipedia, 2016).

It should be noted that the following two parameters are important characteristics of any power plant: 1) overall (gross) or net efficiency ${ }^{1}$ of a plant (for details, see Table 4a,b); and 2) Capacity factor ${ }^{2}$ of a plant (for details, see Table 5 ).

Usually, thermal- and nuclear-power plants operate semi-continuously, because of a high capital cost and low operating costs. The relative costs of electrical energy generated by any system are not only dependent on building capital costs and operating expenses, but also dependent on the capacity factor. The higher the capacity factor the better, as generating costs fall proportionally. However, some renewable-energy sources with exception of large hydroelectric power plants can have significantly lower capacity factors compared to those of thermal- and nuclear-power plants (Handbook, 2016; Pioro and Duffey, 2015).

Therefore, thermal power plants, NPPs and large hydro power plants are considered as the basis for any electrical grid as concentrated and reliable sources of electricity generation. Also, NPPs have essentially negligible operating emissions of carbon dioxide into atmosphere compared to alternate thermal plants. Due to that this source of energy is considered as the most viable one for electrical generation for the next 50 - 100 years (Handbook, 2016; Pioro and Duffey, 2015) (see Tables 6 and 7).

\footnotetext{
${ }^{1}$ Gross efficiency of a unit during a given period of time is the ratio of the gross electrical energy generated by a unit to the energy consumed during the same period by the same unit. The difference between gross and net efficiencies is internal needs for electrical energy of a power plant, which might be not so small ( $5 \%$ or even more).

${ }^{2}$ The net capacity factor of a power plant is the ratio of the actual output of a power plant over a period of time (usually, during a year) and its potential output if it had operated at full nameplate capacity the entire time. To calculate the capacity factor, the total amount of energy a plant produced during a period of time should be divided by the amount of energy the plant would have produced at the full capacity. Capacity factors vary significantly depending on the type of a plant.
} 


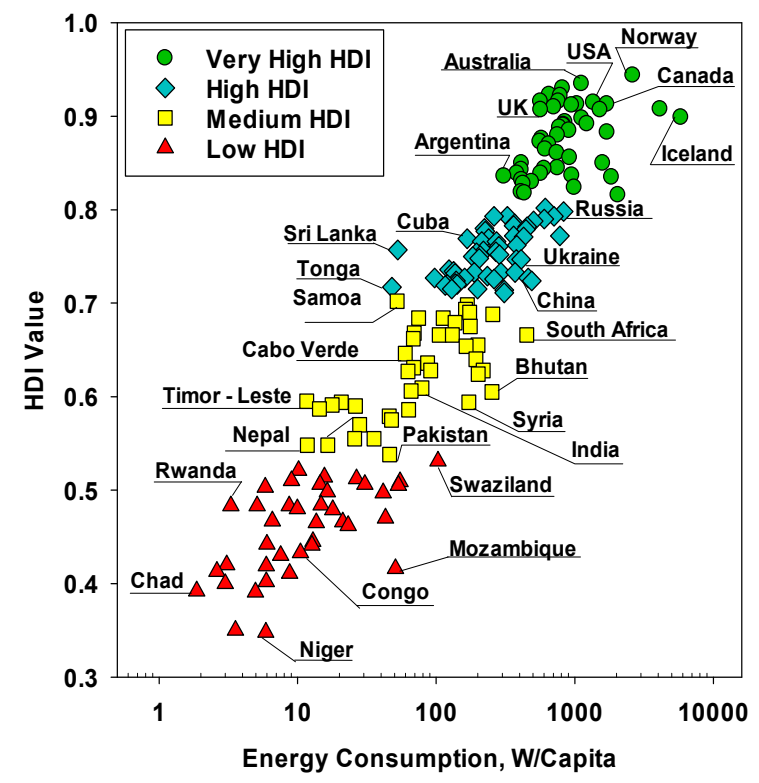

(a)

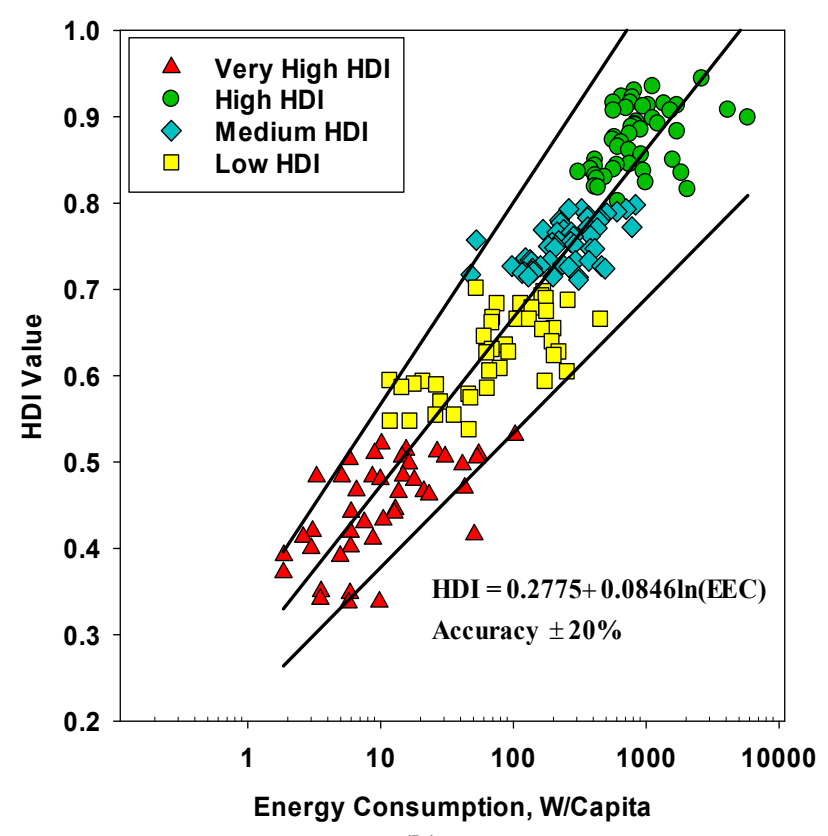

(b)

Fig. 1 Effect of Electrical-Energy Consumption (EEC) on Human Development Index (HDI) for all countries of the world (based on data from Handbook (2016); Human Development Report (2013); The World Fact Book (2013)): (a) graph with selected countries shown and (b) HDI correlation (in general, the HDI correlation might be an exponential rise to maximum (1), but based on the current data it is a straight line in regular - logarithmic coordinates)

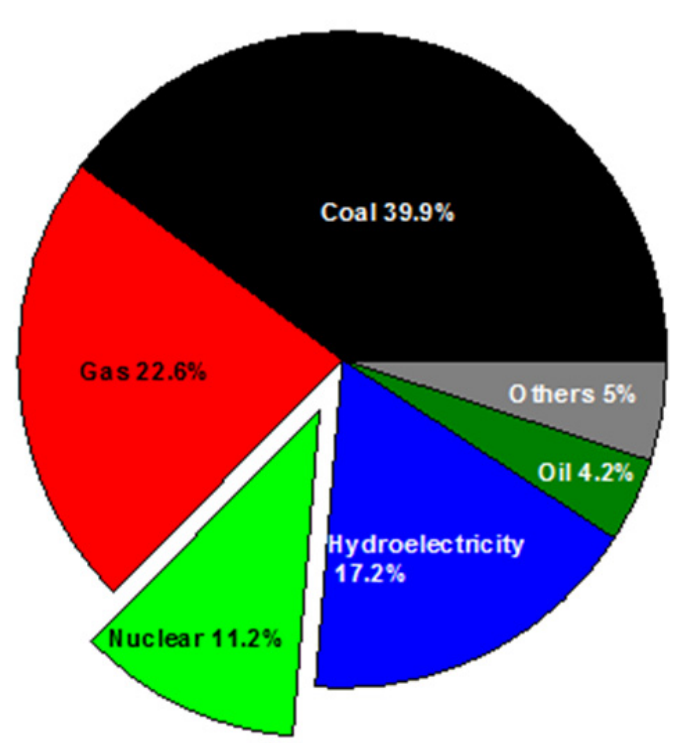

(a) World: Population 7,257 millions; EEC 19,710 TW h/year or 310 W/Capita; HDI 0.711 or HDI Rank 103

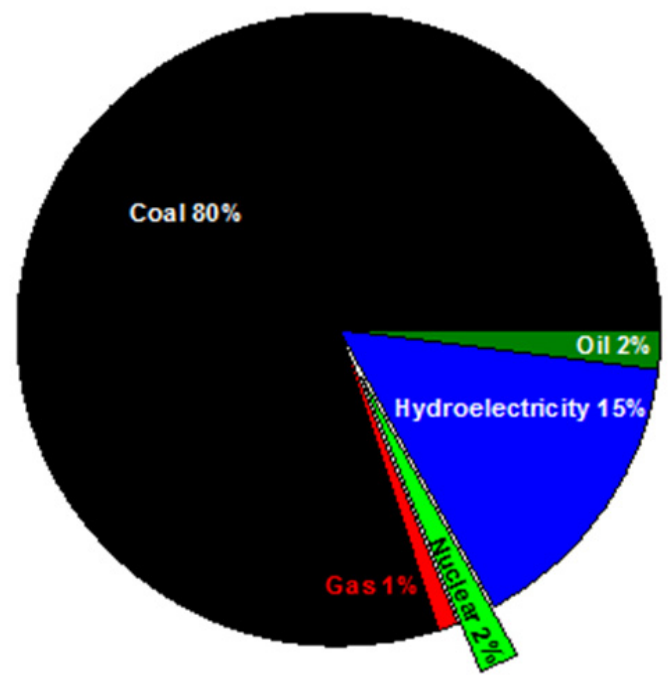

(b) China: Population 1,368 millions; EEC 5,523 TW h/year or 461 W/Capita; HDI 0.727 or HDI Rank 90 


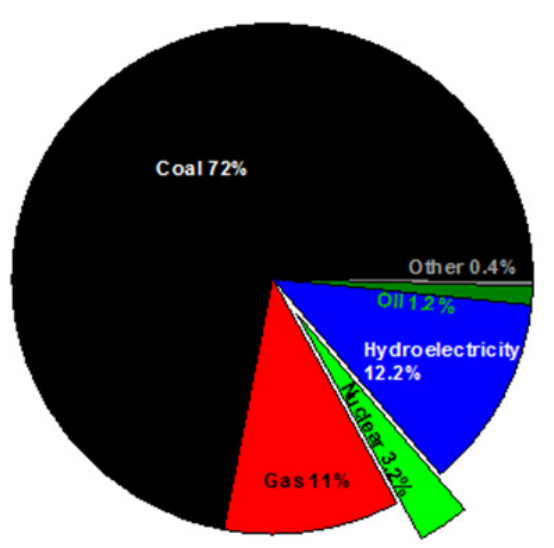

(c) India: Population 1,252 millions; EEC $865 \mathrm{TW}$ h/year or 79 W/Capita; HDI 0.609 or HDI Rank 130

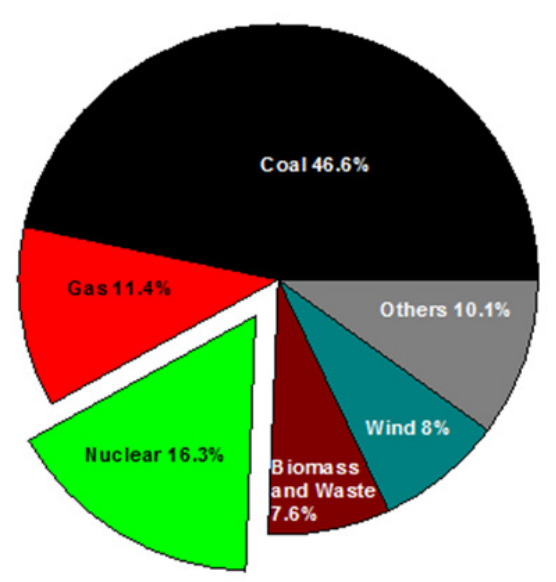

(e) Germany: Population 81 millions; EEC 540 TW h/year or 762 W/Capita; HDI 0.916 or HDI Rank 6

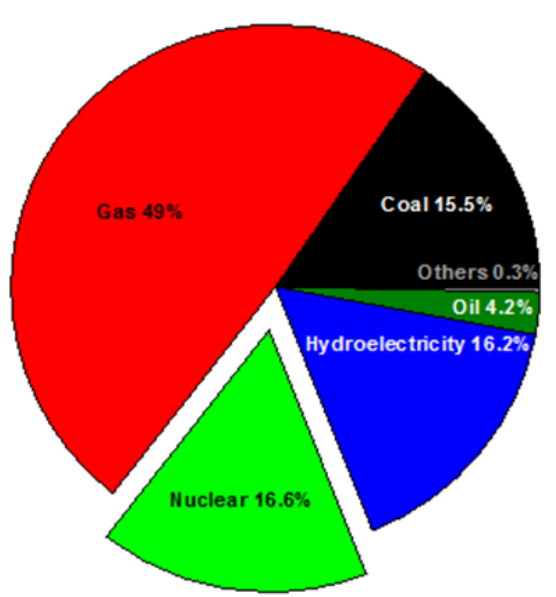

(g) Russia: Population 142 millions; EEC 1,037 TW h/year or 831 W/Capita; HDI 0.798 or HDI Rank 50

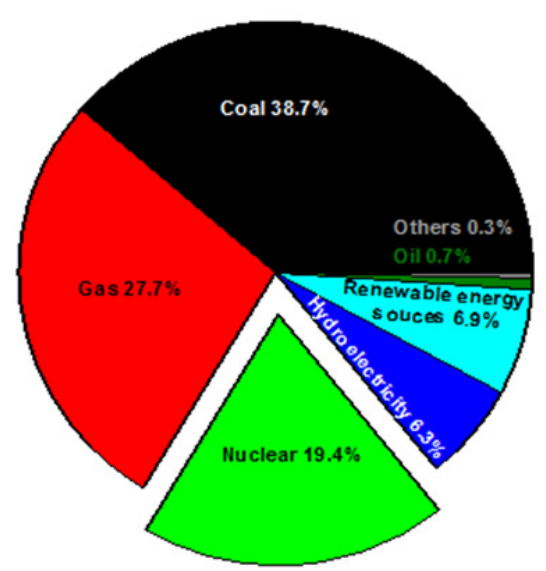

(d) USA: Population 321 millions; EEC 3,832 TW h/year or 1,360 W/Capita; HDI 0.915 or HDI Rank 8; Renewables (6.9\%): Wind (4.4\%); Biomass (1.7\%); Geothermal (0.4\%); and Solar $(0.4 \%)$

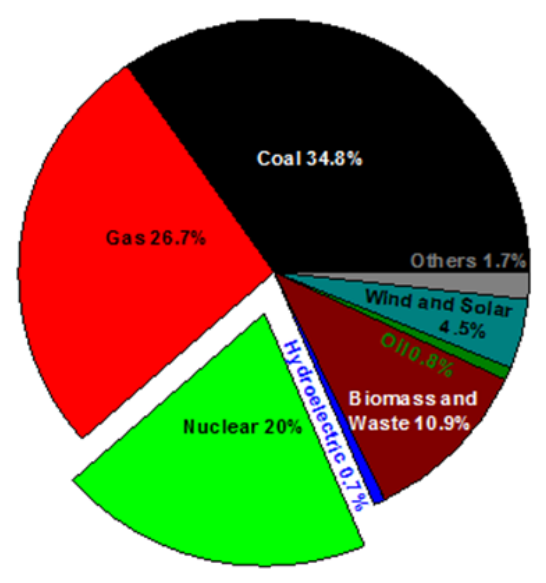

(f) UK: Population 64 millions; EEC 319 TW h/year or 568 W/Capita; HDI 0.907 or HDI Rank 14

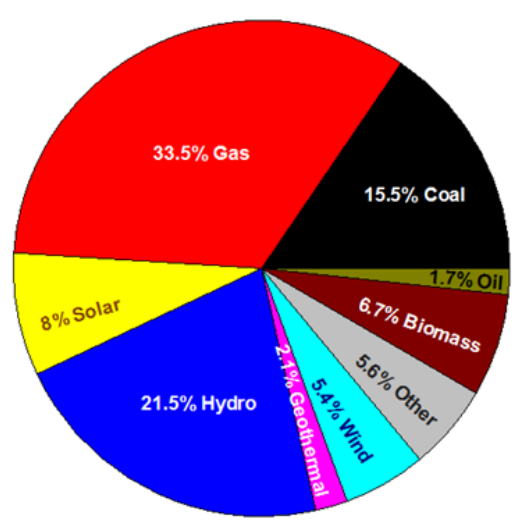

(h) Italy: Population 62 millions; EEC 303 TW h/year or 559 W/Capita; HDI 0.873 or HDI Rank 27 


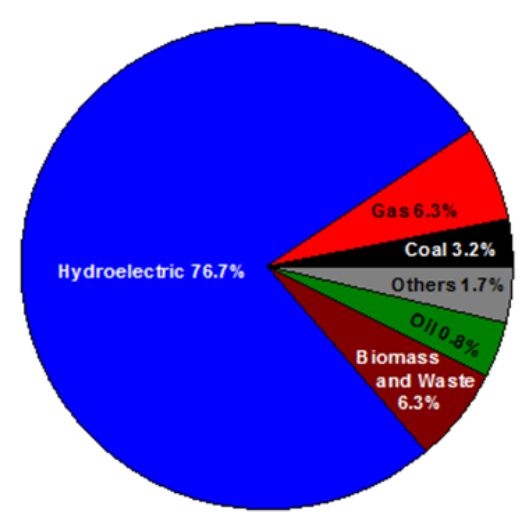

(i) Brazil: Population 204 millions; EEC 484 TW h/year or 270 W/Capita; HDI 0.755 or HDI Rank 75

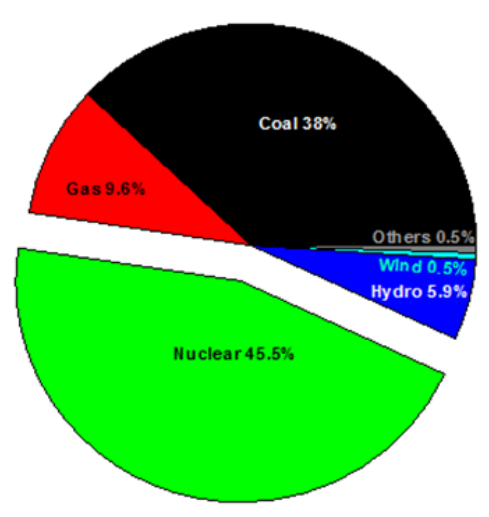

(k) Ukraine: Population 44 millions; EEC $160 \mathrm{TW}$ h/year or 410 W/Capita; HDI 0.747 or HDI Rank 81

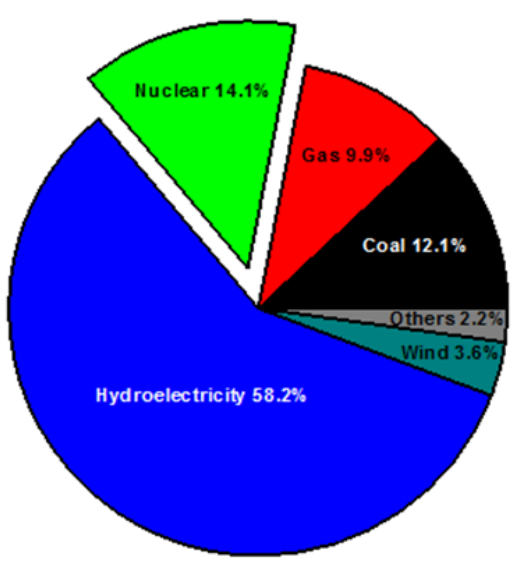

(j) Canada: Population 35 millions; EEC 525 TW h/year or 1,706 W/Capita; HDI 0.913 or HDI Rank 9

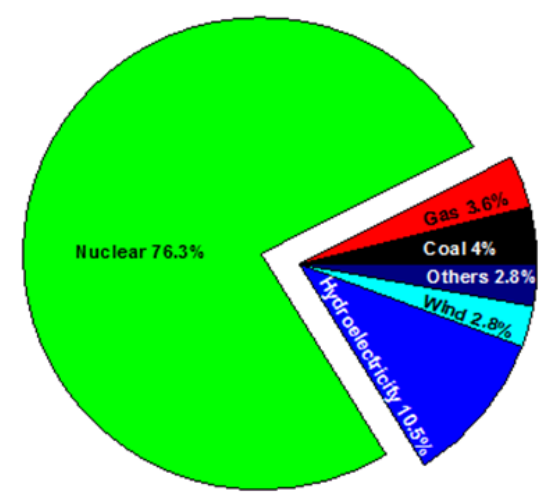

(l) France: Population 67 millions; EEC 451 TW h/year or 773 W/Capita; HDI 0.888 or HDI Rank 22

Fig. 2 Electricity generation by source in the world and selected countries (data from 2010 - 2014 presented here just for reference purposes) Handbook (2016)

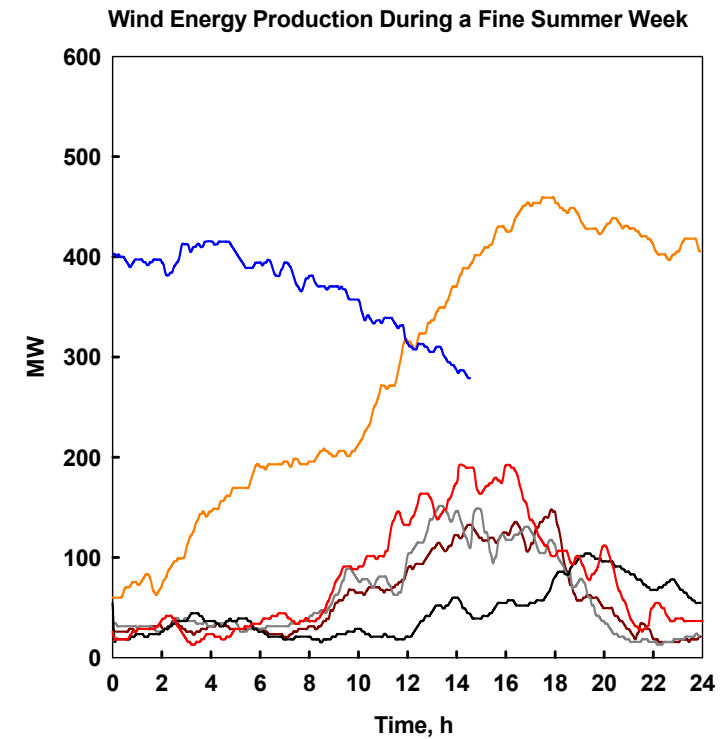

Fig. 3. Power generated by 650-MWel wind turbines in the Western Part of Denmark (based on data from wdww.wiki.windpower.org). Shown summer week (6 days, i.e., various color lines) of wind-power generation

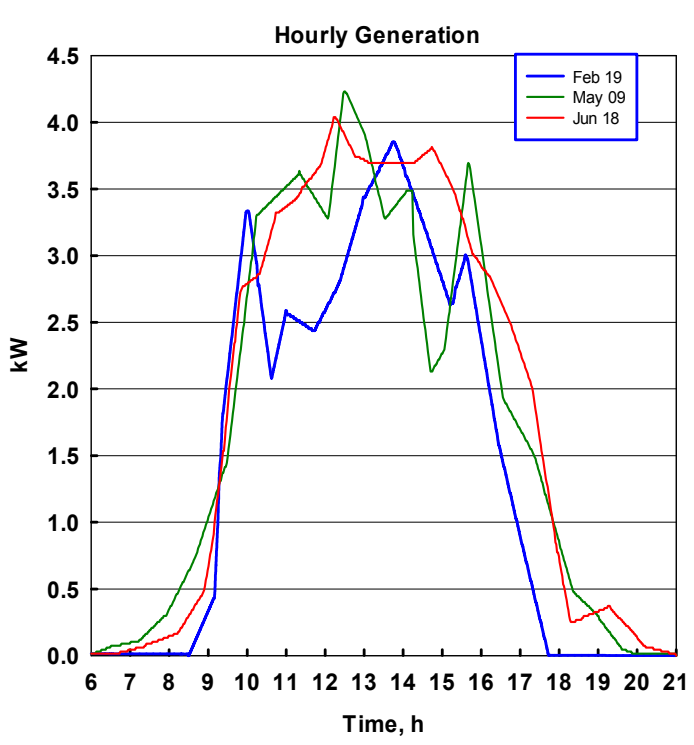

Fig. 4. Power generated by photovoltaic system in New York State (USA) (based on data from www.burningcutlery.com/solar): Shown three mostly sunny days: February 19th; May 9th and June 18th 
Eleven top power plants of the world by installed capacity ${ }^{3}$ (Wikipedia, 2017)

\begin{tabular}{|c|c|c|c|c|c|c|}
\hline No & Plant & Country & $\begin{array}{c}\text { Capacity } \\
\text { MW }_{\mathrm{el}}\end{array}$ & $\begin{array}{c}\text { Average annual } \\
\text { generation } \\
\text { TWh }\end{array}$ & $\begin{array}{l}\text { Capacity } \\
\text { factor, } \%\end{array}$ & $\begin{array}{l}\text { Plant } \\
\text { type }\end{array}$ \\
\hline 1 & Three Gorges Dam & China & 22,500 & 98.8 & 50 & Hydro \\
\hline 2 & Itaipu Dam & Brazil/Paraguay & 14,000 & 98.6 & 72 & Hydro \\
\hline 3 & Xiluodu & China & 13,860 & 57.1 & 47 & Hydro \\
\hline 4 & Guri Dam & Venezuela & 10,200 & - & - & Hydro \\
\hline 5 & Tucurui Dam & Brazil & 8,370 & - & - & Hydro \\
\hline 6 & Kashiwazaki-Kariwa & Japan & 7,965 & - & - & Nuclear \\
\hline 7 & Grand Coulee Dam & USA & 6,809 & 21.0 & 35 & Hydro \\
\hline 8 & Longtan Dam & China & 6,426 & 18.7 & 33 & Hydro \\
\hline 9 & $\begin{array}{l}\text { Sayano- } \\
\text { Shushenskaya }\end{array}$ & Russia & 6,400 & 24.0 & 43 & Hydro \\
\hline 10 & Bruce NPP & Canada & 6,231 & 45.6 & 83 & Nuclear \\
\hline 11 & Krasnoyarsk Dam & Russia & 6,000 & 23.0 & 44 & Hydro \\
\hline
\end{tabular}

Currently, Bruce Nuclear Power Plant (NPP) is the largest fully-operating nuclear plant in the world.

Table 3

Largest operating power plants of the world (based on installed capacity) by energy source (Wikipedia, 2017)

\begin{tabular}{|c|c|c|c|c|}
\hline Rank & Plant & Country & Capacity, $\mathbf{M W}_{\mathrm{el}}$ & Plant type \\
\hline 1 & Three Gorges Dam Power Plant & China & 22,500 & Hydro \\
\hline 2 & Bruce NPP & Canada & 6,231 & Nuclear \\
\hline 3 & Taichung Power Plant & Taiwan & 5,780 & Coal \\
\hline 4 & Shoaiba & S. Arabia & 5,600 & Fuel oil ${ }^{*}$ \\
\hline 5 & Surgut-2 & Russia & 5,597 & Natural gas \\
\hline 6 & Eesti Power Plant & Estonia & 1,615 & Oil shale \\
\hline 7 & Shatura Power Plant & Russia & 1,500 & Peat ${ }^{*}$ \\
\hline 7 & Gansu & China & 5,160 & Wind \\
\hline 8 & Ivanpah Solar Power Facility & USA & 392 & Solar (thermal) \\
\hline 9 & The Geysers & USA & 1,808 & Geothermal \\
\hline 10 & Drax Power Plant & UK & 660 & Biofuel $^{*}$ \\
\hline 11 & Sihwa Lake Tidal Power Plant & S. Korea & 254 & Tidal \\
\hline 12 & Topaz & USA & 550 & Solar (PV**) \\
\hline 13 & $\begin{array}{l}\text { Vasavi Basin Bridge Diesel Power } \\
\text { Plant }\end{array}$ & India & 200 & Diesel \\
\hline 14 & Islay Limpet & UK & 0.5 & Marine (wave) ${ }^{* * *}$ \\
\hline
\end{tabular}

" It should be noted that actually, some thermal power plants use multi-fuel options, for example, Surgut-2 (15\% natural gas), Shatura (peat $-11.5 \%$, natural gas $-78 \%$, fuel oil $-6.8 \%$ and coal $-3.7 \%$ ), Alholmens Kraft (primary fuel - biomass, secondary - peat and tertiary - coal) power plants. $* * \mathrm{PV}-$ PhotoVoltaic. $* * *$ Currently, not in operation anymore.

\footnotetext{
${ }^{3}$ Information provided in Table 2 is considered to be correct within some timeframe. New units can be added and/or some units can be out of service; for example, currently, i.e., May of 2017, the Kashiwazaki-Kariwa NPP is out of service after the earthquake and tsunami disaster and as the result - the severe accident at the Fukushima NPP in Japan in March of 2011.
} 
Table $4 \mathrm{a}$

Typical ranges of thermal efficiencies (gross) of modern thermal power plants (Handbook, 2016; Pioro and Kirillov, 2013b)

\begin{tabular}{|c|c|c|}
\hline No & Thermal Power Plant & $\begin{array}{l}\text { Gross Eff., } \\
\quad \%\end{array}$ \\
\hline 1 & $\begin{array}{l}\text { Combined-cycle power plant (combination of Brayton gas-turbine cycle (fuel - natural gas } \\
\text { or LNG; combustion-products parameters at the gas-turbine inlet: } T_{\mathrm{in}} \approx 1650^{\circ} \mathrm{C} \text { ) and Rankine } \\
\text { steam-turbine cycle (steam parameters at the turbine inlet: } T_{\mathrm{in}} \approx 620^{\circ} \mathrm{C}\left(T_{\mathrm{cr}}=374^{\circ} \mathrm{C}\right) \text { ) }\end{array}$ & Up to 62 \\
\hline 2 & $\begin{array}{l}\text { Supercritical-pressure coal-fired power plant (Rankine-cycle steam inlet turbine parameters: } \\
\left.P_{\mathrm{in}} \approx 23.5-38 \mathrm{MPa}\left(P_{\mathrm{cr}}=22.064 \mathrm{MPa}\right), T_{\mathrm{in}} \approx 540-625^{\circ} \mathrm{C}\left(T_{\mathrm{cr}}=374^{\circ} \mathrm{C}\right) \text { and } T_{\text {reheat }} \approx 540-625^{\circ} \mathrm{C}\right)\end{array}$ & Up to 55 \\
\hline 3 & $\begin{array}{l}\text { Internal-combustion-engine generators (Diesel cycle and Otto cycle with natural gas as a } \\
\text { fuel) }\end{array}$ & Up to 50 \\
\hline 4 & $\begin{array}{l}\text { Subcritical-pressure coal-fired power plant (older plants) (Rankine-cycle steam: } P_{\text {in }} \approx 17 \\
\left.\mathrm{MPa}, T_{\mathrm{in}} \approx 540^{\circ} \mathrm{C}\left(T_{\mathrm{cr}}=374^{\circ} \mathrm{C}\right) \text { and } T_{\text {reheat }} \approx 540^{\circ} \mathrm{C}\right)\end{array}$ & Up to 43 \\
\hline 5 & $\begin{array}{l}\text { Concentrated-solar thermal power plants with heliostats, solar receiver (heat exchanger) on a } \\
\text { tower and molten-salt heat-storage system: Molten-salt maximum temperature is about } \\
565^{\circ} \mathrm{C} \text {, Rankine steam-turbine power cycle used }\end{array}$ & Up to 20 \\
\hline
\end{tabular}

Table $4 \mathrm{~b}$

Typical ranges of thermal efficiencies (gross) of modern NPPs (Handbook, 2016; Pioro and Kirillov, 2013c)

\begin{tabular}{|c|c|c|}
\hline No & Nuclear Power Plant & $\begin{array}{l}\text { Gross Eff., } \\
\quad \%\end{array}$ \\
\hline 1 & $\begin{array}{l}\text { Carbon-dioxide-cooled reactor NPP (Generation-III) (reactor coolant: } P=4 \mathrm{MPa} \& \\
T=290-650^{\circ} \mathrm{C} \text {; steam: } P=16.7 \mathrm{MPa}\left(T_{\text {sat }}=351^{\circ} \mathrm{C} \text { and } T_{\mathrm{cr}}=374^{\circ} \mathrm{C}\right) \& T_{\text {in }}=538^{\circ} \mathrm{C} \text {; reheat: } P=4.1 \\
\left.\mathrm{MPa} \& T_{\text {in }}=538^{\circ} \mathrm{C}\right)\end{array}$ & Up to 42 \\
\hline 2 & $\begin{array}{l}\text { Sodium-cooled fast reactor NPP (Generation-IV) (steam: } P=14 \mathrm{MPa}\left(T_{\mathrm{sat}}=337^{\circ} \mathrm{C}\right) \& T_{\mathrm{in}}=505^{\circ} \mathrm{C} \\
\left.\text { and reheat: } P=2.45 \mathrm{MPa} \& T_{\mathrm{in}}=505^{\circ} \mathrm{C}\right)\end{array}$ & Up to 40 \\
\hline 3 & $\begin{array}{l}\text { Pressurized Water Reactor NPP* (Generation-III }+ \text {, to be implemented within next } 1-10 \text { years) } \\
\text { (reactor coolant: } P=15.5 \mathrm{MPa} \& T_{\mathrm{out}}=327^{\circ} \mathrm{C} \text {; steam: } P=7.8 \mathrm{MPa} \& T_{\mathrm{in}}=T_{\mathrm{sat}}=293^{\circ} \mathrm{C} \text { and reheat) }\end{array}$ & Up to 38 \\
\hline 4 & $\begin{array}{l}\text { Pressurized Water Reactor NPP* (Generation-III, current fleet) (reactor coolant: } P=15.5 \mathrm{MPa} \\
\& T_{\text {out }}=329^{\circ} \mathrm{C} \text {; steam: } P=6.9 \mathrm{MPa} \& T_{\mathrm{in}}=T_{\text {sat }}=285^{\circ} \mathrm{C} \text { and reheat) }\end{array}$ & Up to 36 \\
\hline 5 & $\begin{array}{l}\text { Boiling Water Reactor NPP* (Generation-III, current fleet) (direct cycle) }\left(P_{\text {in }}=7.2 \mathrm{MPa} \&\right. \\
T_{\text {in }}=T_{\text {sat }}=288^{\circ} \mathrm{C} \text { and reheat) }\end{array}$ & Up to 34 \\
\hline 6 & $\begin{array}{l}\text { RBMK NPP*(boiling, pressure-channel) }(\text { Generation-III, current fleet })(\text { direct cycle })\left(P_{\text {in }}=6.46\right. \\
\left.\text { MPa \& } T_{\text {in }}=T_{\text {sat }}=280^{\circ} \mathrm{C} \text {; reheat: } P=0.29 \mathrm{MPa} \& T_{\text {reheat }}=263^{\circ} \mathrm{C}\right)\end{array}$ & Up to 32 \\
\hline 7 & $\begin{array}{l}\text { Pressurized Heavy Water Reactor NPP* (Generation-III, current fleet) (reactor coolant: } P=11 \\
\mathrm{MPa} \& T=260-310^{\circ} \mathrm{C} \text {; steam: } P=4.6 \mathrm{MPa} \& T_{\mathrm{in}}=T_{\text {sat }}=259^{\circ} \mathrm{C} \text { and reheat) }\end{array}$ & Up to 32 \\
\hline
\end{tabular}

Note to table: 1) All NPPs with water-cooled reactors use only Rankine cycle with saturated steam at the inlet of a turbine and steam reheat, which uses primary saturated steam as the heating medium. 
Table 5

Average (typical) capacity factors of various power plants (listed here just for reference purposes) (Handbook, 2016)

\begin{tabular}{|c|c|c|c|c|}
\hline No & Power Plant type & Location & Year & Capacity factor, $\%$ \\
\hline \multirow[t]{2}{*}{1} & \multirow[t]{2}{*}{ Nuclear } & USA & 2010 & 91 \\
\hline & & UK & 2011 & 66 \\
\hline \multirow[t]{2}{*}{2} & \multirow[t]{2}{*}{ Combined-cycle } & USA & 2009 & 42 \\
\hline & & UK & 2011 & 48 \\
\hline \multirow[t]{2}{*}{3} & \multirow[t]{2}{*}{ Coal-fired } & USA & 2009 & 64 \\
\hline & & UK & 2011 & 42 \\
\hline \multirow[t]{3}{*}{4} & \multirow[t]{3}{*}{ Hydroelectric $^{4}$} & USA and UK & 2011 & 40 \\
\hline & & World (average) & - & 44 \\
\hline & & World (range) & - & $10-99$ \\
\hline \multirow[t]{2}{*}{5} & \multirow[t]{2}{*}{ Wind } & UK & 2011 & 30 \\
\hline & & World & 2008 & $20-40$ \\
\hline 6 & Wave & Portugal & - & 20 \\
\hline 7 & Concentrated-solar thermal & $\begin{array}{l}\text { USA California } \\
\text { Spain }\end{array}$ & $\begin{array}{l}- \\
-\end{array}$ & $\begin{array}{l}21 \\
75\end{array}$ \\
\hline \multirow[t]{3}{*}{8} & \multirow[t]{3}{*}{ Photovoltaic (PV) solar } & USA Arizona & 2008 & 19 \\
\hline & & USA Massachusetts & - & $12-15$ \\
\hline & & UK & 2011 & $5-8$ \\
\hline 9 & Concentrated-solar PV & Spain & - & 12 \\
\hline
\end{tabular}

Table 6

Number of nuclear-power reactors in operation and forthcoming as per March 2017 (Nuclear News, 2017) and before the Japan earthquake and tsunami disaster (March 2011; Nuclear News, 2011)

\begin{tabular}{|c|c|c|c|c|c|c|c|}
\hline \multirow[t]{2}{*}{ No } & \multirow[t]{2}{*}{$\begin{array}{c}\text { Reactor type } \\
\text { (Some details on reactors) }\end{array}$} & \multicolumn{2}{|c|}{ No. of units } & \multicolumn{2}{|c|}{$\begin{array}{l}\text { Installed capacity, } \\
\qquad \mathbf{G W}_{\mathrm{el}}\end{array}$} & \multicolumn{2}{|c|}{$\begin{array}{l}\text { Forthcoming } \\
\text { units }\end{array}$} \\
\hline & & $\begin{array}{c}\text { As of } \\
\text { March } \\
2017\end{array}$ & $\begin{array}{l}\text { Before } \\
\text { March } \\
2011\end{array}$ & $\begin{array}{l}\text { As of } \\
\text { March } \\
2017\end{array}$ & $\begin{array}{l}\text { Before } \\
\text { March } \\
2011\end{array}$ & $\begin{array}{c}\text { No. of } \\
\text { units }\end{array}$ & $\mathbf{G W}_{\mathrm{e}}$ \\
\hline 1 & $\begin{array}{l}\text { Pressurized Water Reactors (PWRs) } \\
\text { (largest group of nuclear reactors in the world }-64 \% \text { ) }\end{array}$ & $287 \uparrow$ & 268 & $271 \uparrow$ & 248 & 77 & 83.5 \\
\hline 2 & $\begin{array}{l}\text { Boiling Water Reactors (BWRs) or Advanced BWRs } \\
\left(2^{\text {nd }} \text { largest group of reactors in the world }-18 \% \text {; ABWRs - }\right. \\
\text { the only ones Generation-III+ operating reactors })\end{array}$ & $78 \downarrow$ & 92 & $76 \downarrow$ & 84 & 6 & 8 \\
\hline 3 & $\begin{array}{l}\text { Pressurized Heavy Water Reactors (PHWRs) } \\
\left(3^{\text {rd }} \text { largest group of reactors in the world }-11 \% \text {; mainly }\right. \\
\text { CANDU-reactor type })\end{array}$ & $48 \downarrow$ & 50 & $24 \downarrow$ & 25 & 9 & 6.0 \\
\hline 4 & $\begin{array}{l}\text { Advanced Gas-cooled Reactors (AGRs) (UK, } 14 \text { reactors); } \\
\text { (all these } \mathrm{CO}_{2} \text {-cooled reactors will be shut down in the } \\
\text { nearest future and will not be built again) }(3 \%)\end{array}$ & $14 \downarrow$ & 18 & $7.7 \downarrow$ & 9 & $1^{1}$ & 0.2 \\
\hline
\end{tabular}

\footnotetext{
${ }^{4}$ Capacity factors depend significantly on a design, size and location (water availability) of a hydroelectric power plant. Small plants built on large rivers will always have enough water to operate at a full capacity.
} 


\begin{tabular}{|c|c|c|c|c|c|c|c|}
\hline \multirow[t]{2}{*}{ No } & \multirow[t]{2}{*}{$\begin{array}{c}\text { Reactor type } \\
\text { (Some details on reactors) }\end{array}$} & \multicolumn{2}{|c|}{ No. of units } & \multicolumn{2}{|c|}{$\begin{array}{l}\text { Installed capacity, } \\
\text { GW }_{\text {el }}\end{array}$} & \multicolumn{2}{|c|}{$\begin{array}{l}\text { Forthcoming } \\
\text { units }\end{array}$} \\
\hline & & $\begin{array}{c}\text { As of } \\
\text { March } \\
2017\end{array}$ & $\begin{array}{l}\text { Before } \\
\text { March } \\
2011\end{array}$ & $\begin{array}{l}\text { As of } \\
\text { March } \\
2017\end{array}$ & $\begin{array}{l}\text { Before } \\
\text { March } \\
2011\end{array}$ & $\begin{array}{c}\text { No. of } \\
\text { units }\end{array}$ & $G W_{\text {el }}$ \\
\hline 5 & $\begin{array}{l}\text { Light-water, Graphite-moderated Reactors (LGRs) } \\
\text { (Russia, } 11 \text { RBMKs and } 4 \text { EGPs; these pressure-channel } \\
\text { boiling-water-cooled reactors will be shut down in the nearest } \\
\text { future and will not be built again) }(3 \%)\end{array}$ & 15 & 15 & 10 & 10 & 0 & 0 \\
\hline 6 & $\begin{array}{l}\text { Liquid-Metal Fast-Breeder Reactors (LMFBRs) } \\
\text { (Russia, SFR - BN-600; only one Generation-IV operating } \\
\text { reactor) }\end{array}$ & $2 \uparrow$ & 1 & $1.3 \uparrow$ & 0.6 & 3 & 0.6 \\
\hline & In total & 444 & 444 & $391 \uparrow$ & 378 & 96 & 98 \\
\hline
\end{tabular}

Table 7

Number of nuclear-power reactors by nation (11 nations with the largest installed nuclear-power capacities) as per March of 2017 (Nuclear News, 2017) and before the Japan earthquake and tsunami disaster (March of 2011)

(Nuclear News, 2011)

\begin{tabular}{|c|c|c|c|c|c|c|}
\hline \multirow[t]{2}{*}{ No } & \multirow[t]{2}{*}{ Nation } & \multicolumn{2}{|c|}{ No. of units (PWRs/BWRs) } & \multicolumn{2}{|c|}{ Installed capacity, $\mathbf{G W}_{\mathrm{el}}$} & \multirow{2}{*}{$\begin{array}{c}\text { Changes in number of reactors } \\
\text { from March } 2011\end{array}$} \\
\hline & & As of March 2017 & $\begin{array}{c}\text { Before } \\
\text { March } \\
2011\end{array}$ & $\begin{array}{c}\text { As of } \\
\text { March } \\
2017\end{array}$ & $\begin{array}{c}\text { Before } \\
\text { March 2011 }\end{array}$ & \\
\hline 1 & USA & $99(65 / 34)$ & 104 & 101 & 103 & Decreased by 5 reactors \\
\hline 2 & France & $58(58 /-)$ & 58 & 63 & 63 & No changes \\
\hline 3 & Japan $^{*}$ & $42(19 / 23)$ & 54 & 40 & 47 & $\downarrow$ Decreased by 12 reactors \\
\hline 4 & China & $35\left(33 /-/ 2^{3}\right)$ & 13 & 31 & 10 & $\uparrow$ Increased by 22 reactors \\
\hline 5 & Russia & $34\left(17 /-/ 15^{1} / 2^{2}\right)$ & 32 & 25 & 23 & $\uparrow$ Increased by 2 reactors \\
\hline 6 & S. Korea & $25\left(21 /-/ 4^{3}\right)$ & 20 & 23 & 18 & $\uparrow$ Increased by 5 reactors \\
\hline 7 & Canada & $19\left(-/-/ 19^{3}\right)$ & 22 & 13 & 15 & $\downarrow$ Decreased by 3 reactors \\
\hline 8 & Ukraine & $15(15 /-)$ & 15 & 13 & 13 & No changes \\
\hline 9 & Germany & $8(6 / 2)$ & 17 & 11 & 20 & $\downarrow$ Decreased by 9 reactors \\
\hline 10 & Sweden & $10(7 / 3)$ & 10 & 9.7 & 9.3 & No changes \\
\hline 11 & UK & $15\left(1 /-/ 14^{4}\right)$ & 19 & 8.9 & 10 & $\downarrow$ Decreased by 4 reactors \\
\hline
\end{tabular}

Arrows mean decrease or increase in a number of reactors.

${ }^{1}$ No of LGRs; ${ }^{2}$ LMFBRs; ${ }^{3}$ PHWRs; ${ }^{4}$ AGRs.

Figure 5 shows a number of nuclear-power reactors of the world put into commercial operation vs. years and age of operating reactors (Handbook, 2016). Five reactors have been put into operation in 1969, i.e., they operate for more than 46 years. It is clear from this diagram that the Chernobyl NPP accident has tremendous negative impact on the world nuclear-power industry, which lasts for decades. And currently, we have additional negative impact of the Fukushima Daiichi NPP accident. Figure 6 shows possible scenarios of nuclear-power development in the world. In general, in spite of all current advances into nuclear power, modern NPPs have the following deficiencies:

1) Generate radioactive wastes;

2) Have relatively low thermal efficiencies, especially, water-cooled NPPs (up to 1.6 times lower than that for modern advanced thermal power plants; 
3) Risk of radiation release during severe accidents; and

4) Production of nuclear fuel is not an environment-friendly process.

Therefore, all these deficiencies should be addressed in next generation - Generation IV NPPs (for details, see Table 8.

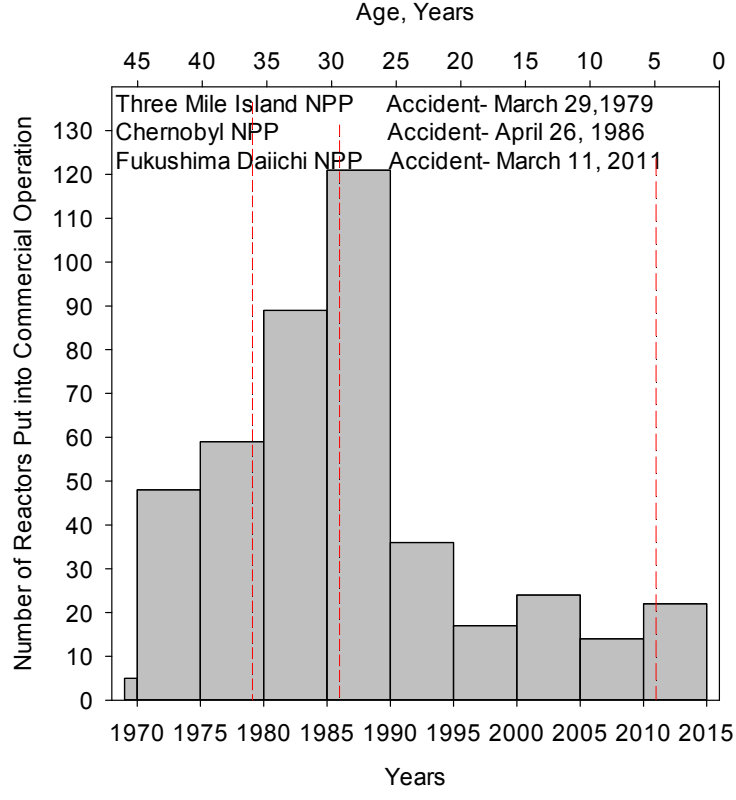

Fig. 5. Number of nuclear-power reactors of the world put into commercial operation vs. years and age of operating reactors (Handbook, 2016)

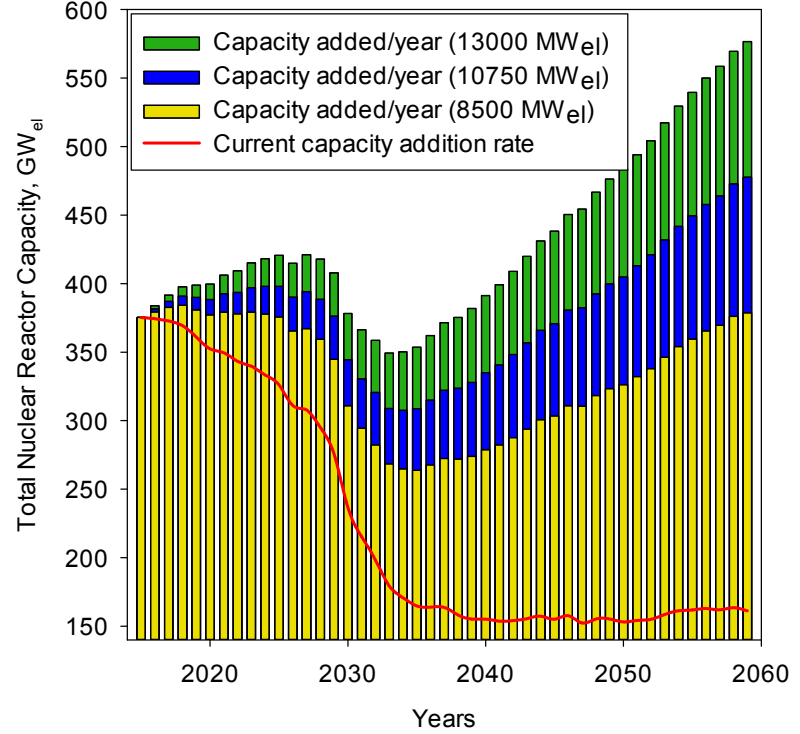

Fig. 6. Possible scenarios of nuclear-power development in the world (based on assumption that average term of reactors' operation is 45 years)

Table 8

Estimated ranges of thermal efficiencies (gross) of Generation-IV NPP concepts (Generation IV concepts are listed according to thermal efficiency decrease) (shown here just for reference purposes) (Handbook, 2016)

\begin{tabular}{|c|c|c|}
\hline No & Nuclear Power Plant & $\begin{array}{c}\text { Gross Eff., } \\
\%\end{array}$ \\
\hline 1 & $\begin{array}{l}\text { Very High Temperature Reactor (VHTR) NPP (reactor coolant }- \text { helium: } P=7 \mathrm{MPa} \text { and } \\
T_{\text {in }} / T_{\text {out }}=640 / 1000^{\circ} \mathrm{C} \text {; primary power cycle }- \text { direct Brayton gas-turbine cycle; possible back-up } \\
\text { - indirect Rankine steam cycle). }\end{array}$ & $\geq 55$ \\
\hline 2 & $\begin{array}{l}\text { Gas-cooled Fast Reactor (GFR) or High Temperature Reactor (HTR) NPP (reactor coolant - } \\
\text { helium: } P=9 \mathrm{MPa} \text { and } T_{\text {in }} / T_{\text {out }}=490 / 850^{\circ} \mathrm{C} \text {; primary power cycle }- \text { direct Brayton gas-turbine } \\
\text { cycle; possible back-up - indirect Rankine steam cycle). }\end{array}$ & $\geq 50$ \\
\hline 3 & $\begin{array}{l}\text { SuperCritical Water-cooled Reactor (SCWR) NPP (one of Canadian concepts; reactor coolant } \\
\text { - light water: } P=25 \mathrm{MPa} \text { and } T_{\mathrm{in}} / T_{\text {out }}=350 / 625^{\circ} \mathrm{C}\left(T_{\mathrm{cr}}=374^{\circ} \mathrm{C}\right) \text {; direct cycle; high-temperature } \\
\text { steam superheat: } T_{\text {out }}=625^{\circ} \mathrm{C} \text {; possible back-up - indirect supercritical-pressure Rankine steam } \\
\text { cycle with high-temperature steam superheat). }\end{array}$ & $45-50$ \\
\hline 4 & $\begin{array}{l}\text { Molten Salt Reactor (MSR) NPP (reactor coolant - sodium-fluoride salt with dissolved } \\
\text { uranium fuel: } T_{\text {out }}=700 / 800^{\circ} \mathrm{C} \text {; primary power cycle }- \text { indirect supercritical-pressure carbon- } \\
\text { dioxide Brayton gas-turbine cycle; possible back-up - indirect Rankine steam cycle). }\end{array}$ & $\sim 50 \%$ \\
\hline
\end{tabular}




\begin{tabular}{|c|c|c|}
\hline No & Nuclear Power Plant & $\begin{array}{c}\text { Gross Eff., } \\
\%\end{array}$ \\
\hline 5 & $\begin{array}{l}\text { Lead-cooled Fast Reactor (LFR) NPP (Russian design Brest-300: reactor coolant }- \text { liquid lead: } \\
P \approx 0.1 \mathrm{MPa} \text { and } T_{\text {in }} / T_{\text {out }}=420 / 540^{\circ} \mathrm{C} ; \text { primary power cycle }- \text { indirect supercritical-pressure } \\
\text { Rankine steam cycle: } P_{\text {in }} \approx 24.5 \mathrm{MPa}\left(P_{\mathrm{cr}}=22.064 \mathrm{MPa}\right) \text { and } T_{\text {in }} / T_{\text {out }}=340 / 520^{\circ} \mathrm{C}\left(T_{\mathrm{cr}}=374^{\circ} \mathrm{C}\right) ; \\
\text { high-temperature steam superheat; possible back-up in some other countries }- \text { indirect } \\
\text { supercritical-pressure carbon-dioxide Brayton gas-turbine cycle). }\end{array}$ & $\sim 43$ \\
\hline 6 & $\begin{array}{l}\text { Sodium-cooled Fast Reactor (SFR) NPP (Russian design BN-600: reactor coolant }- \text { liquid } \\
\text { sodium (primary circuit): } P \approx 0.1 \mathrm{MPa} \text { and } T_{\text {in }} / T_{\text {out }}=380 / 550^{\circ} \mathrm{C} \text {; liquid sodium (secondary } \\
\text { circuit): } T_{\text {in }} / T_{\text {out }}=320 / 520^{\circ} \mathrm{C} \text {; primary power cycle }- \text { indirect Rankine steam cycle: } P_{\text {in }} \approx 14.2 \\
\mathrm{MPa}\left(T_{\text {sat }} \approx 337^{\circ} \mathrm{C}\right) \text { and } T_{\text {in max }}=505^{\circ} \mathrm{C}\left(T_{\mathrm{cr}}=374^{\circ} \mathrm{C}\right) \text {; steam superheat: } P \approx 2.45 \mathrm{MPa} \text { and } \\
T_{\text {in }} / T_{\text {out }}=246 / 505^{\circ} \mathrm{C} \text {; possible back-up in some other countries - indirect supercritical-pressure } \\
\text { carbon-dioxide Brayton gas-turbine cycle). }\end{array}$ & $\sim 40$ \\
\hline
\end{tabular}

Interaction between various electricity-generating sources inside one system (electrical grid) can be illustrated based on that in the Province of Ontario ${ }^{5}$ (Canada) (Handbook, 2016). Figure 7a shows installed capacity and Figure 7b - electricity generation by energy source in Ontario (Canada) in 2012. Figure 7a shows that in Ontario major installed capacities in 2012 were nuclear (34\%), gas (26\%), hydro (22\%), coal (8\%), and renewables (mainly wind) (8\%). However, the electricity (see Figure 7 b) was mainly generated by nuclear $(56 \%)$, hydro (22\%), natural gas (10\%), renewables (mainly wind) (5\%), and coal (2\%).

Figure 8a shows power generated by various energy sources in Ontario (Canada) on June 19, 2012 (a peak power on hot summer day, when major air-conditioning was required) and corresponding to that Figure 8b shows capacity factors of these energy sources. Figure 8 shows that electricity that day from midnight till 3.00 in the morning was mainly generated by nuclear, hydro, gas, wind, "other" and coal. After 3.00 in the morning, wind power fell due to Mother Nature, but electricity consumption started to rise. Therefore, "fast-response" gas-fired power plants and, later, hydro and coal-fired power plants plus "other" power plants started to increase electricity generation to compensate for both decreasing in wind power and increasing demand for electricity. After 18.00 in the evening, energy consumption slightly dropped in the province, and at the same time, wind power started to be increased by Mother Nature. Therefore, gas-fired, hydro and "other" power plants decreased energy generation accordingly. After 22.00 o'clock in the evening, energy consumption dropped even more. Therefore, coal-fired power plants with the most emissions decreased abruptly their electricity generation followed by gas-fired and hydro-power plants.

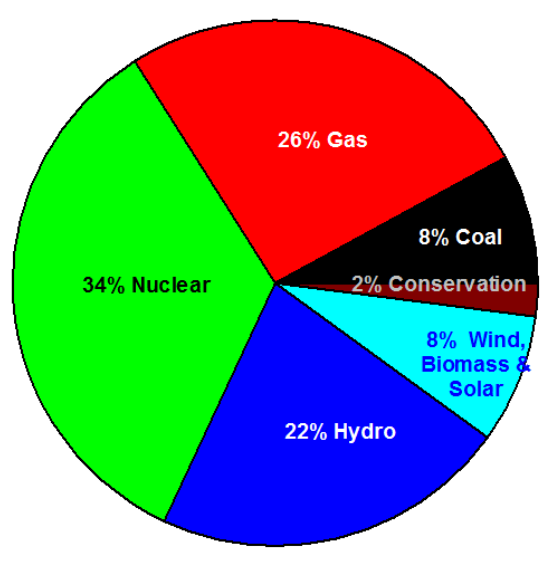

(a)

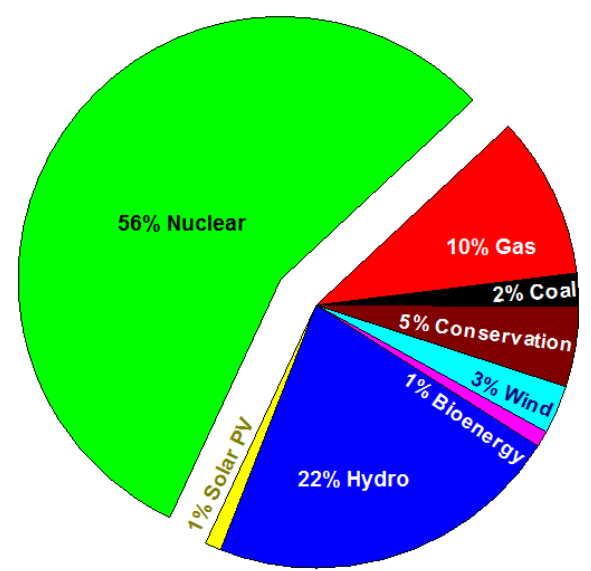

(b)

Fig. 7. Installed capacity (a) and electricity generation (b) by energy source in Ontario (Canada), 2012-2013 (based on data from Ontario Power Authority: http://www.powerauthority.on.ca and Ontario's Long-Term Energy Plan)

\footnotetext{
${ }^{5}$ Population: $\sim 14$ million (2016); Area: 1.076 million km²; Capital: Toronto (location $43.65^{\circ}$ North) (for comparison, location of Nice, France $43.71^{\circ}$ North).
} 


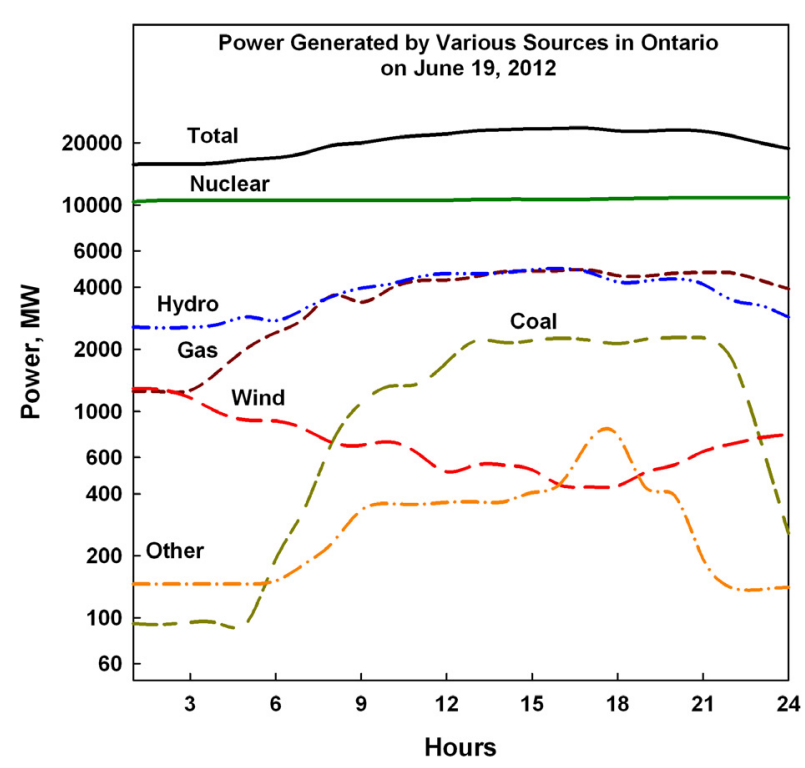

(a)

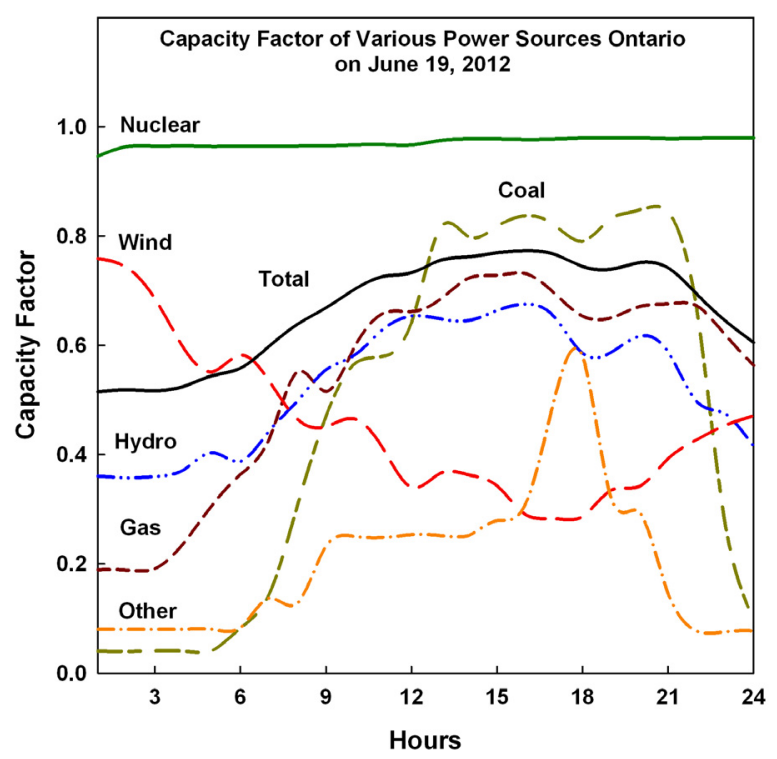

(b)

Fig. 8. Power generated (a) and capacity factors (b) of various energy sources in Ontario (Canada) on June 19, 2012 (based on data from http://ieso.ca/imoweb/marketdata/genEnergy.asp) (shown here just for reference purposes)

However, currently, the Province of Ontario (Canada) has completely eliminated coal-fired power plants from the electrical grid (Handbook, 2016). Some of them were closed, others - converted to natural gas. Figure 9a shows installed capacity, and Figure 9b - electricity generation by energy source in the Province of Ontario (Canada) in 2015. Figure 9a shows that in Ontario major installed capacities in 2015 were nuclear (38\%), gas (29\%), hydro (25\%), and renewables (mainly wind) (8\%). However, electricity (see Figure 9b) was mainly generated by nuclear $(60 \%)$, hydro (24\%), natural gas $(8.7 \%)$, and renewables (mainly wind) $(4.9 \%)$.

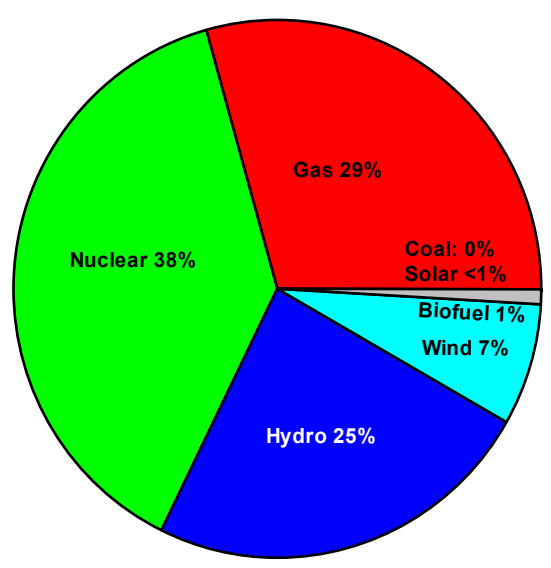

(a)

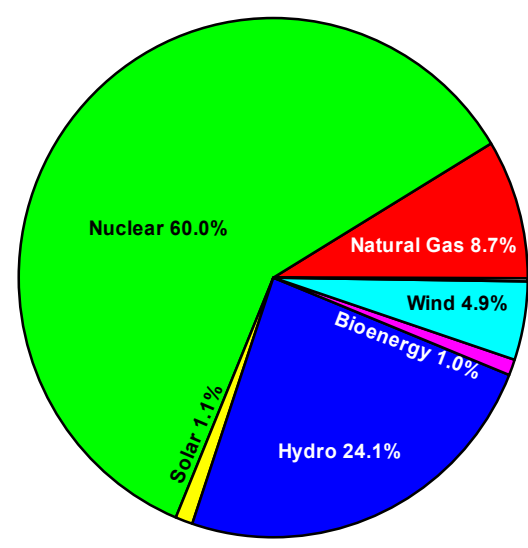

(b)

Fig. 9. Installed capacity (a) and electricity generation (b) by energy source in Ontario (Canada), 2014-2015 (based on data from Ontario Energy Board: http://www.ontarioenergyboard.ca/ and Ontario Energy Report http://www.ontarioenergyreport.ca/)

Figure 10a,b shows power generated by various energy sources in Ontario (Canada) on June 17, 2015 and corresponding to that capacity factors of various energy sources. Figure 10 shows that electricity that day from midnight till 3.00 in the morning was mainly generated by nuclear, hydro, gas, wind, and biofuel. After 3.00, biofuel power plants increased slightly electricity generation followed by hydro and gas-fired power plants. Also, at the same time, wind-power plants started to generate slightly more electricity due to Mother Nature. However, after 7.00 wind power started to fluctuate and, eventually, decreased quite significantly. After 6.00 in the morning, solar-power plants 
started to generate some electricity ${ }^{6}$. During a day, hydro, gas-fired and biofuel power plants had variable electricity generation to compensate changes in consumption of electrical energy and variations in generating electricity with wind and solar power plants. After 21.00 in the evening, energy consumption started to drop in the province, and at the same time, wind power increased by Mother Nature. Therefore, gas-fired, hydro and biofuel power plants decreased energy generation accordingly. In both cases, i.e., June 19 of 2012 and June 17 of 2015, NPPs operated at about 100\% of installed capacity providing reliable basic power to the grid.

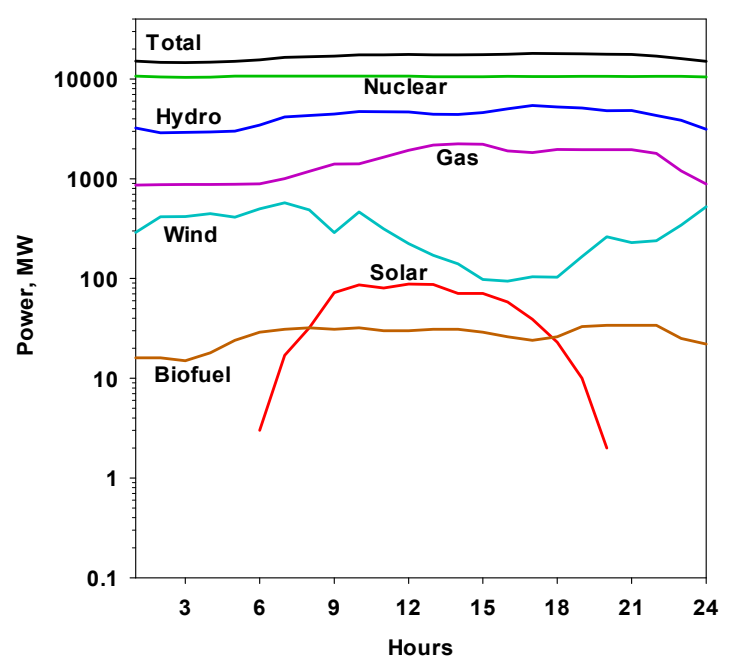

(a)

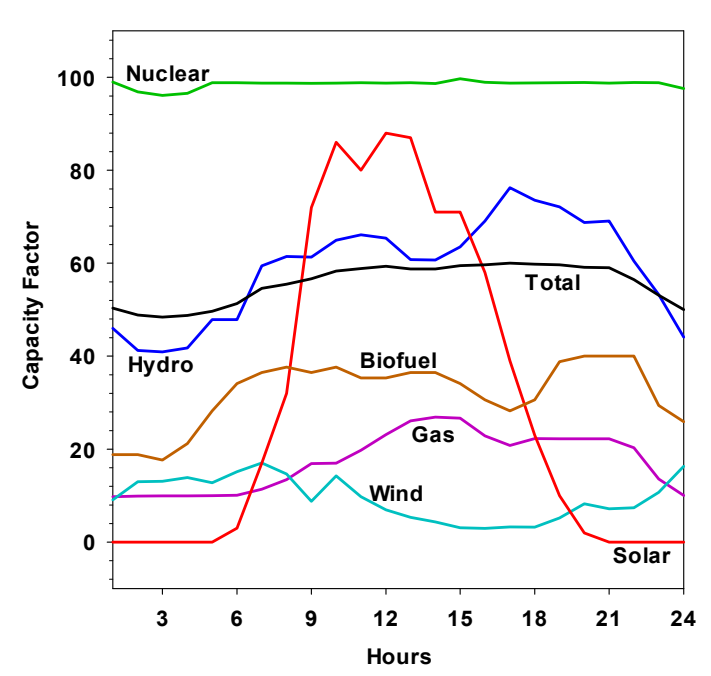

(b)

Fig. 10. Power generated (a) and capacity factors (b) of various energy sources in Ontario (Canada) on June 17, 2015

(based on data from http://ieso.ca/imoweb/marketdata/genEnergy.asp) (shown here just for reference purposes)

These examples show clearly that any grid that includes NPPs and/or renewable-energy sources must also include "fast-response" power plants such as gas- and/or coal-fired and/or large hydro-power plants. This is due not only to diurnal and seasonal peaking of demand, but also the diurnal and seasonal variability of supply. Thus, for any given market, the generating mix and the demand cycles must be matched 24/7/365, independent of what sources are used, and this requires flexible control and an appropriate mix of base-load and peaking plants.

Also, it should be noted here that having a large percent of variable power sources mainly such as wind and solar, and other, i.e., which generating capacity depends on Mother Nature, an electrical grid can collapse due to significant and unpredicted power instabilities! In addition, the following detrimental factors are usually not considered during estimation of variable power-sources costs: 1) costs of fast-response power plants with service crews on site $24 / 7$ as a back-up power and 2) faster amortization / wear of equipment of fast-response plants.

\section{CURRENT AND FUTURE STATUS OF UKRAINIAN POWER INDUSTRY}

Ukraine has about 42 million people and is the largest European country by a territory with exception of Russia. Ukraine consumes about $182 \mathrm{TW}$ h/year electrical energy from various sources (mainly from nuclear - 45.5 \% and coal $-38 \%$ (for details, see Figure 2c)) or has about $461 \mathrm{~W} /$ Capita (see Table 1 and Figure 1). Due to that Ukraine is currently on the $78^{\text {th }}$ place by HDI in the world, which is at the lower end of the second group of countries with High HDI (countries from $43^{\text {rd }}$ and up to $85^{\text {th }}$ places by HDI).

The Ukrainian nuclear-power industry consists of four NPPs with the total of 15 reactors (see Table 9 and Figure 11). Thermodynamic layout of a VVER-1000 NPP is shown in Figure 12. Major parameters of the Russian-design PWRs - VVERs operated in Ukraine are listed in Table 10 and $T-s$ diagram of the VVER-1000 turbine cycle - in Figure 13.

Analysis of the Ukrainian power industry shows that two of these $15^{\text {th }}$ reactors have been built and put into operation in 70-s, ten in 80-s, one in 90-s and just two - in 2004. Also, it should be noted current problems of Ukrainian NPPs, which are: 1) lower capacity factors (around 80\%) compared to those in other countries ( 90\%) (Handbook, 2016); 2) uncertainties with nuclear-fuel supply due to political situation; and 3) service and repairs of relatively old reactors.

\footnotetext{
${ }^{6}$ It should be noted that usually, solar power plants might generate only a small amount of electricity within late fall, winter and early spring compared to that during summer.
} 
Based on an analysis of the world power reactors in terms of their maximum years of operation (currently, the oldest reactors are 45-year old Nuclear News, 2016)) several projections have been made for future of the nuclearpower industry in Ukraine (for details, see Figure 14). Unfortunately, all these projections are quite pessimistic.

There is a possibility that around 2030-2035 the vast majority of the Ukrainian reactors will be shut down, and Ukraine can be left without the basic and vital source of electricity generation.

Table 9

General information on Ukrainian NPPs (http://www.energoatom.kiev.ua/en/)

\begin{tabular}{|c|c|c|c|c|c|}
\hline \multirow{2}{*}{$\begin{array}{l}\text { Ukrainian } \\
\text { NPP }\end{array}$} & \multirow{2}{*}{$\begin{array}{c}\text { Unit } \\
\#\end{array}$} & \multirow[t]{2}{*}{ Reactor } & \multirow{2}{*}{$\frac{\text { Power }}{\text { MW }_{\text {el }}}$} & \multicolumn{2}{|r|}{ Year } \\
\hline & & & & Startup & $\begin{array}{l}\text { Shutdown (based on } \\
\text { original 30-year term) }\end{array}$ \\
\hline \multirow{4}{*}{$\begin{array}{l}\text { Rivne } \\
\text { (planned } 2 \\
\text { more reactors) }\end{array}$} & 1 & VVER- 440 (B-213 type) & 440 & 1977 & 2007 (ext. 2030) \\
\hline & 2 & VVER- 440 (B-213 type) & 440 & 1978 & 2008 (ext. 2030) \\
\hline & 3 & VVER-1000 (model 320) & 1000 & 1989 & 2019 \\
\hline & 4 & VVER-1000 (model 320) & 1000 & 2004 & 2034 \\
\hline \multirow{3}{*}{$\begin{array}{l}\text { South-Ukraine } \\
\text { (planned } 1 \\
\text { more reactor) }\end{array}$} & 1 & VVER-1000 (“'small series”) & 1000 & 1982 & 2012 (ext. 2023) \\
\hline & 2 & VVER-1000 (“small series”) & 1000 & 1985 & 2015 (ext. 2025) \\
\hline & 3 & VVER-1000 (model 320) & 1000 & 1989 & 2019 \\
\hline \multirow{2}{*}{$\begin{array}{l}\text { Khmel'nitsky } \\
\text { (planned } 2 \\
\text { more reactors) }\end{array}$} & 1 & VVER-1000 (model 320) & 1000 & 1984 & 2014 (to be extended) \\
\hline & 2 & VVER-1000 (model 320) & 1000 & 2004 & 2034 \\
\hline \multirow{6}{*}{$\begin{array}{l}\text { Zaporizhzhya } \\
\text { (largest in } \\
\text { Europe) }\end{array}$} & 1 & VVER-1000 (model 320) & 1000 & 1985 & 2015 (to be extended) \\
\hline & 2 & VVER-1000 (model 320) & 1000 & 1986 & 2016 (to be extended) \\
\hline & 3 & VVER-1000 (model 320) & 1000 & 1987 & 2017 \\
\hline & 4 & VVER-1000 (model 320) & 1000 & 1989 & 2019 \\
\hline & 5 & VVER-1000 (model 320) & 1000 & 1989 & 2019 \\
\hline & 6 & VVER-1000 (model 320) & 1000 & 1995 & 2025 \\
\hline
\end{tabular}

Explanations to the Table: ext. - extended till

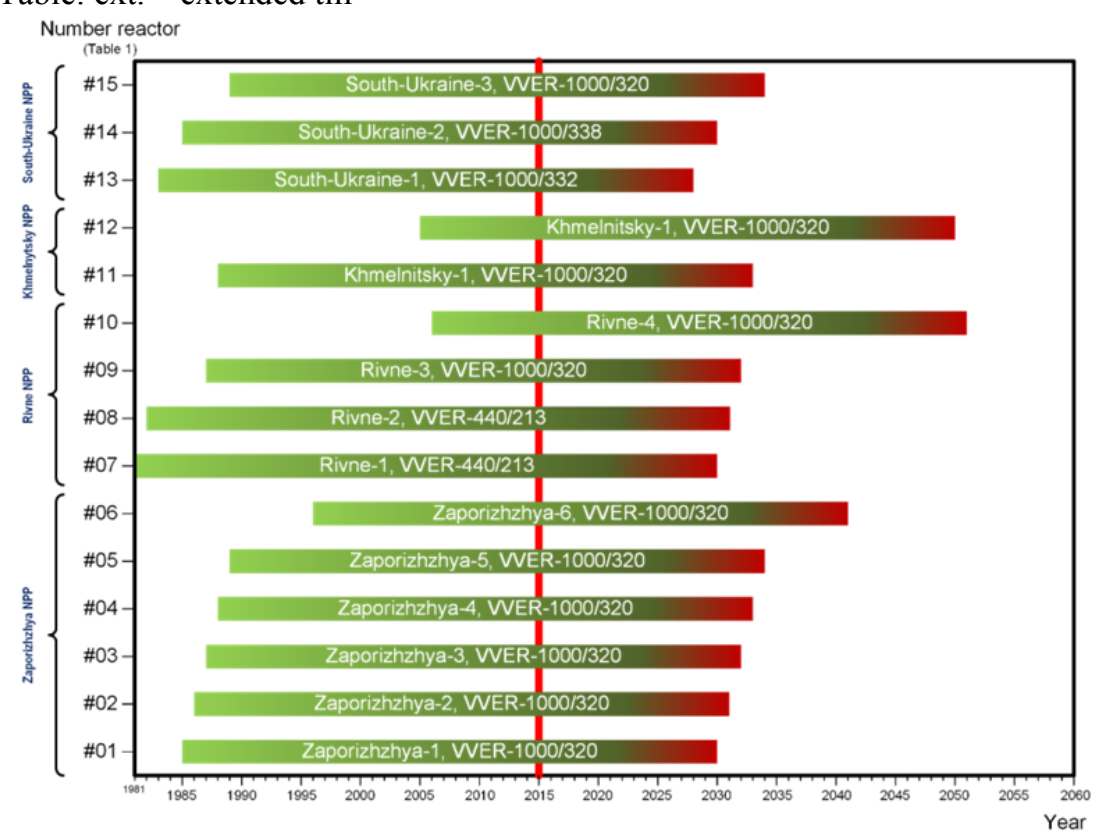

Fig. 11. Shutdown year per each Ukrainian reactor based on possible 45-year extension 


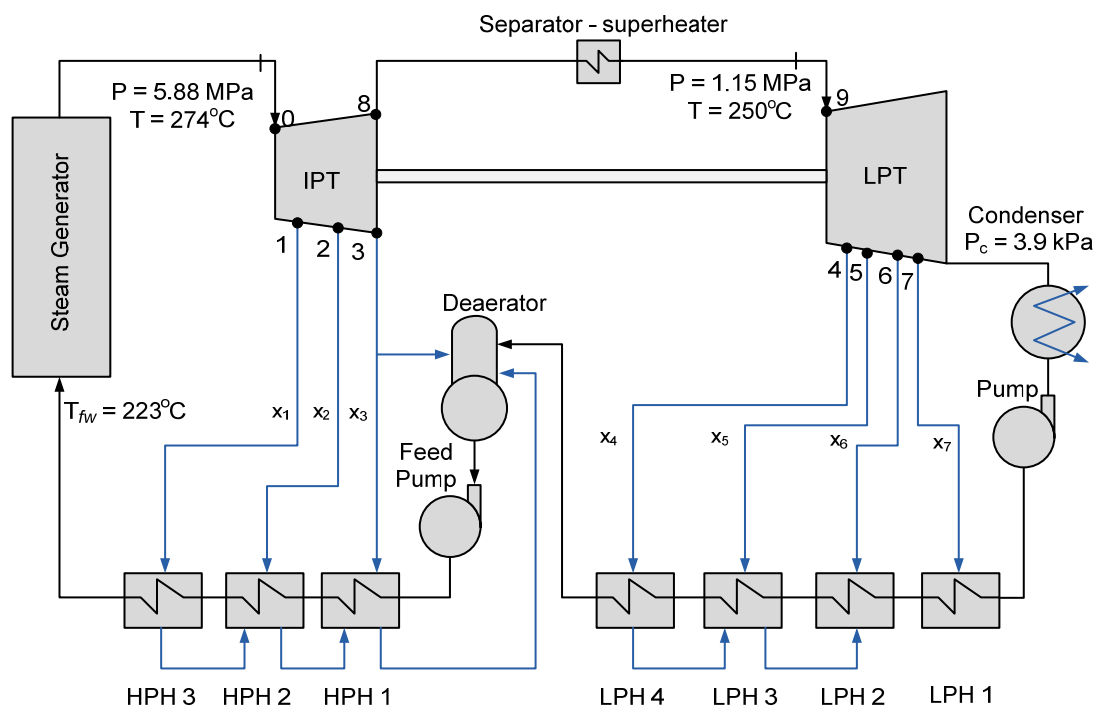

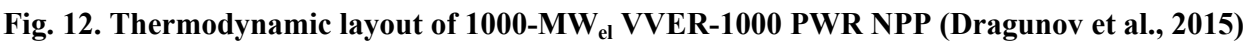

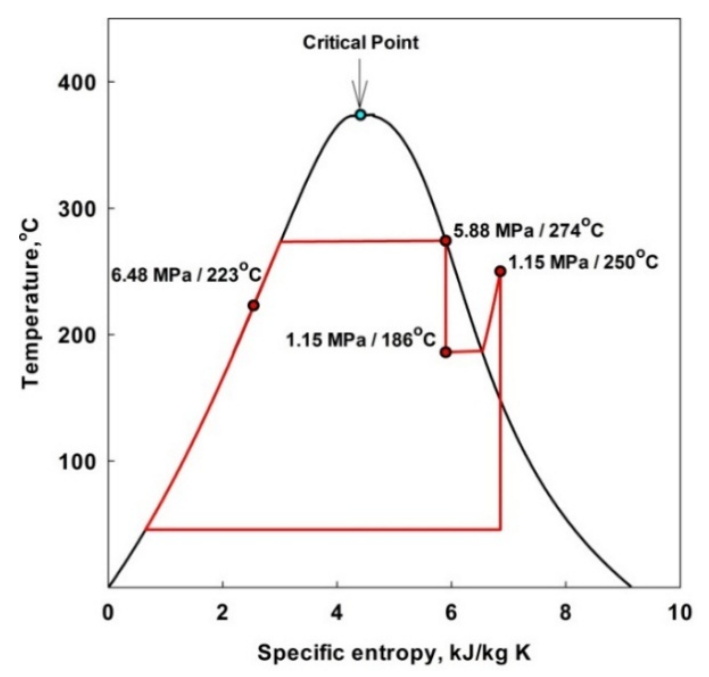

Fig. 13. $T$-s diagram for a VVER-1000 turbine cycle

Major parameters of Ukrainian power reactors (Russian PWR - VVER-type) (Grigor'ev and Zorin, 1988)

\begin{tabular}{|l|c|c|}
\hline \multicolumn{1}{|c|}{ Parameter } & $\begin{array}{c}\text { VVER- } \\
\mathbf{4 4 0}\end{array}$ & $\begin{array}{c}\text { VVER- } \\
\mathbf{1 0 0 0}\end{array}$ \\
\hline Thermal power, $\mathrm{MW}_{\text {th }}$ & 1375 & 3000 \\
\hline Electrical power, MW $\mathrm{el}$ & 440 & 1000 \\
\hline $\begin{array}{l}\text { Thermal efficiency (gross)* } \\
\text { \% }\end{array}$ & 32.0 & 33.0 \\
\hline Coolant pressure, MPa & 12.3 & 15.7 \\
\hline Coolant flow, t/h & 42,600 & 80,000 \\
\hline Coolant temperature, C & $270 / 298$ & $290 / 322$ \\
\hline Average heat flux, MW/m ${ }^{2}$ & 0.378 & 0.545 \\
\hline Steam flow rate, $\mathrm{t} / \mathrm{h}$ & 2700 & 5880 \\
\hline Steam pressure, MPa & 4.6 & 6.48 \\
\hline Steam temperature, C & 258.8 & 280.7 \\
\hline $\begin{array}{l}\text { Core: Diameter/Height, } \\
\text { m/m }\end{array}$ & $3.84 / 11.8$ & $4.5 / 10.9$ \\
\hline $\begin{array}{l}\text { Equivalent diameter of core, } \\
\text { m }\end{array}$ & 2.88 & 3.12 \\
\hline Fuel enrichment (max), \% & 3.6 & 4.3 \\
\hline No. of fuel assemblies & 349 & 151 \\
\hline No. of rods in fuel assembly & 126 & 317 \\
\hline
\end{tabular}

* Thermal efficiencies have been calculated with the IAEA Desalination Thermodynamic Optimization Programme DE-TOP (IAEA, 2013) and compared to the actual ones. 
Analysis of the Ukrainian thermal-power industry shows that 8 large thermal power plants have been built in $60 \mathrm{~s}, 9-$ in $70 \mathrm{~s}$, and 3 in $80 \mathrm{~s}$. Due to this, the vast majority of them quite old and not very efficient plants.

Therefore, Ukraine has to move quickly with building new NPPs with modern reactors. Interesting point here is that Ukraine has its own resources of uranium (up to 800 tonnes per year, which is about $30 \%$ of the country's requirements) and own resources of Zirconium. In addition, there are ten scientific-research institutes related to nuclear science/engineering, nuclear energy, fuel and waste management. Based on that Ukraine might consider as an option to build CANDU reactors, which operate with natural uranium. Through that Ukraine has a possibility to develop its own closed fuel cycle and to have more independent and diversified nuclear-power industry.

Of course, NPPs require to be supported with fast-response thermal power plants, which will cover peaks and drops in electricity consumption per day. Therefore, Ukraine has to move to modern high-efficiency thermal power plants such as combined-cycle power plants (combination of Brayton gas-turbine cycle (fuel - natural gas or liquefied natural gas; combustion-products parameters at the gas-turbine inlet: $T_{\mathrm{in}} \approx 1650^{\circ} \mathrm{C}$ ) and Rankine steam-turbine cycle (steam parameters at the turbine inlet: $T_{\mathrm{in}} \approx 620^{\circ} \mathrm{C}\left(T_{\mathrm{cr}}=374^{\circ} \mathrm{C}\right)$ ) with gross thermal efficiencies of up to $62 \%$ and/or supercritical-pressure coal-fired power plants (Rankine-cycle steam inlet turbine parameters: $P_{\text {in }} \approx 25-38 \mathrm{MPa}$ $\left(P_{\mathrm{cr}}=22.064 \mathrm{MPa}\right), T_{\mathrm{in}} \approx 540-625^{\circ} \mathrm{C}\left(T_{\mathrm{cr}}=374^{\circ} \mathrm{C}\right)$ and $\left.T_{\text {reheat }} \approx 540-625^{\circ} \mathrm{C}\right)$ with thermal efficiencies of up to $55 \%$ (Handbook, 2016; Pioro and Kirillov, 2013).

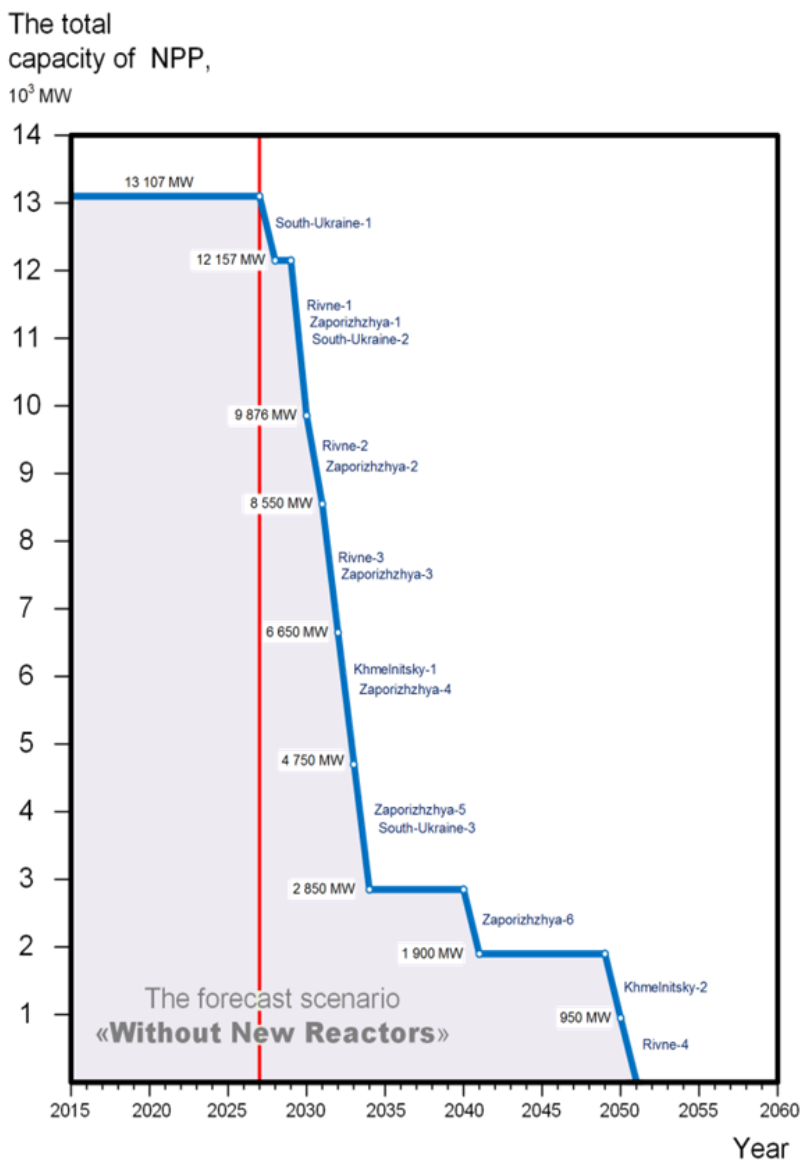

(a)

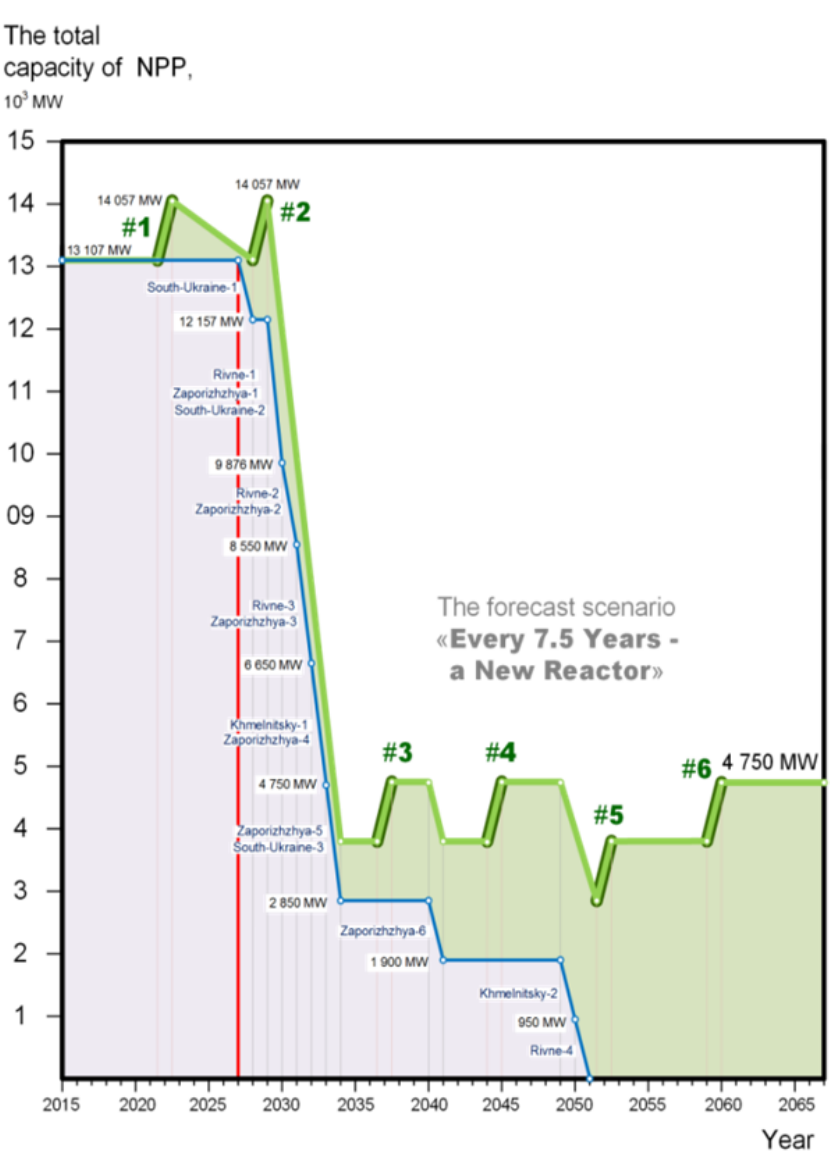

(b) 


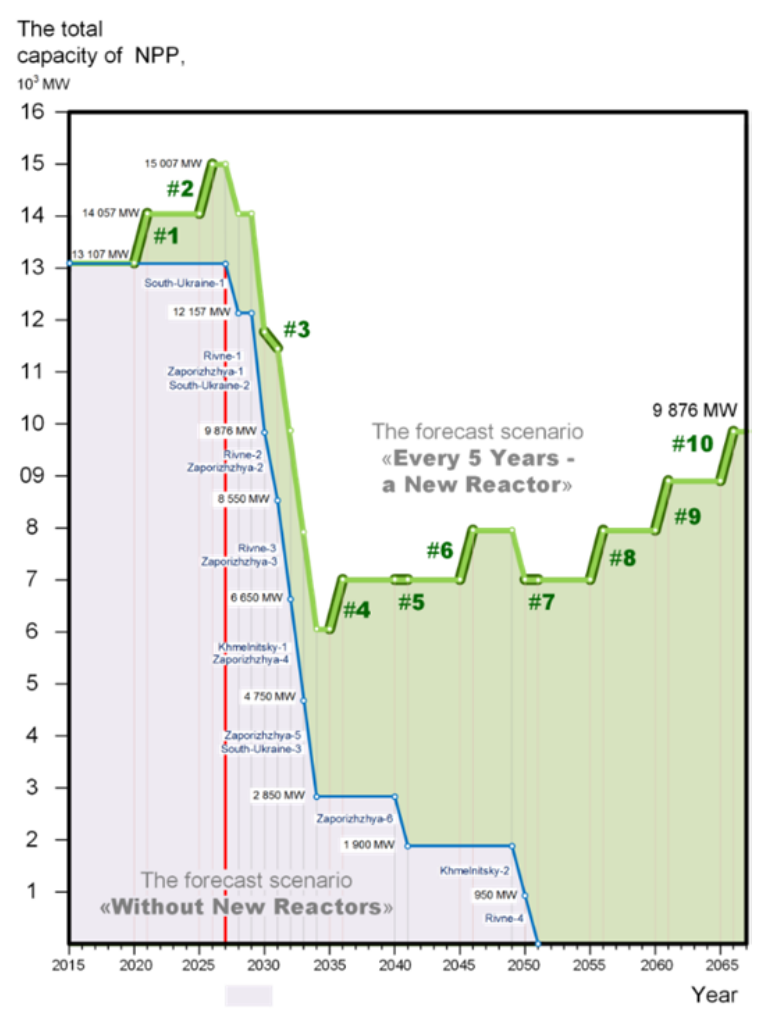

(c)

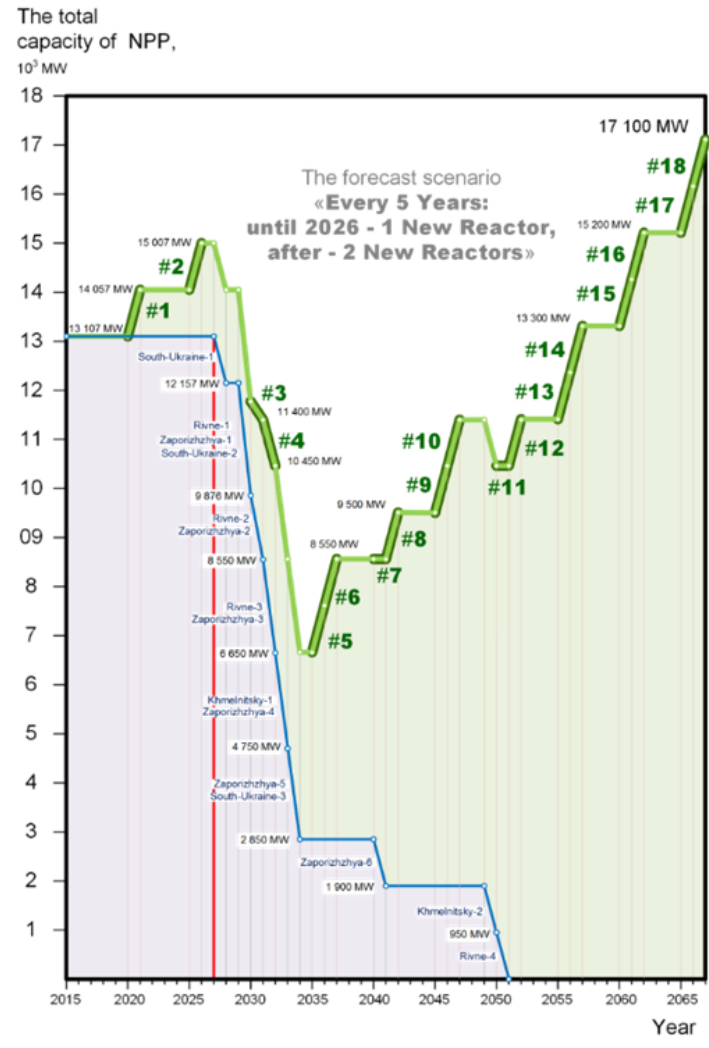

(d)

The total

capacity of NPP,

$10^{3} \mathrm{MW}$

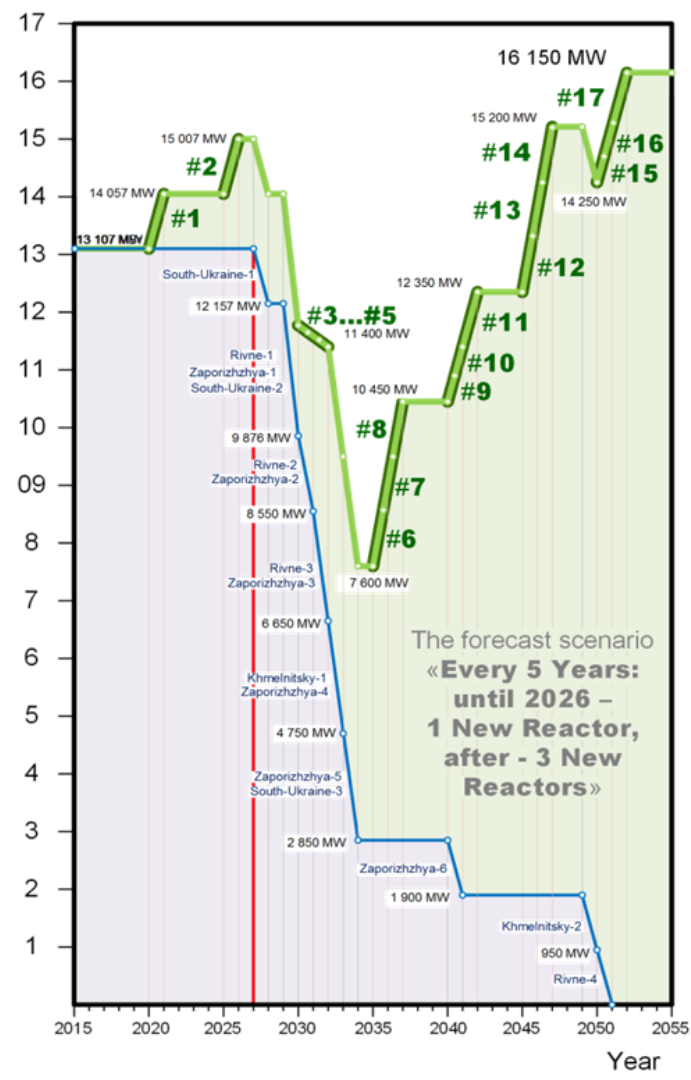

(e)

Fig. 14. Possible scenarios for future of nuclear-power industry in Ukraine 


\section{CONCLUSIONS}

1. Electricity generation is the key factor for advances in industry, agriculture, technology and the level of living. Also, strong power industry with diverse energy sources is very important for country independence.

2. In general, electricity can be generated from: 1) non-renewable energy sources such as coal, natural gas, oil, and nuclear; and 2) renewable energy sources such as hydro, biomass, wind, geothermal, solar, and wave power. However, the major energy sources for electricity generation in the world are: 1$)$ thermal power - primarily using coal $(\sim 40 \%)$ and secondarily - natural gas $(\sim 23 \%) ; 2)$ "large" hydro power plants $(\sim 17 \%)$ and 3$)$ nuclear power from various reactor designs $(\sim 11 \%)$. The rest of the energy sources for electricity generation is from using oil ( $\sim \%)$ and renewable sources such as biomass, wind, geothermal and solar $(\sim 5 \%)$, which have just visible impact in selected countries. In addition, energy sources, such as wind and solar, and some others, like tidal and wavepower, are intermittent from depending on Mother Nature. And cannot be used alone for industrial electricity generation.

3. Currently, Ukraine covers its needs for electricity through using nuclear, thermal and hydro power plants. However, nuclear and thermal power plants are quite old and less efficient than modern NPPs and thermal plants. Also, hydro resources almost used completely.

4. Nuclear power in Ukraine is the most important source of electricity generation in the country. Currently, Ukrainian Nuclear Power Plants (NPPs) generate about $45.5 \%$ of the total electricity followed with coal generation $-38 \%$, gas generation $9.6 \%$ and the rest is based on renewable sources, mainly on hydro power plants $-5.9 \%$. Nuclear-power industry is based on four NPPs (15 Pressurized Water Reactors (PWRs) including the largest one in Europe - Zaporizhzhya NPP with about 6,000 $\mathrm{MW}_{\mathrm{el}}$ gross installed capacity.

5. Two of these 15 reactors have been built and put into operation in 70-s, ten in 80-s, one in 90 -s and just two in 2004. Therefore, based on an analysis of the world power reactors in terms of their maximum years of operation (currently, the oldest reactors are $\sim 45$-year old) several projections have been made for future of the nuclear-power industry in the world and Ukraine. Unfortunately, all these projections are quite pessimistic. There is a possibility that around 2030-2040 the vast majority of the world reactors and Ukrainian reactors will be shut down, and, in particular, Ukraine can be left without the basic and vital source of electricity generation.

6. Therefore, to decrease these negative trends the following measures should be taken: (a) extension of current NPPs terms of operation; (b) building new NPPs with reactors from various nuclear vendors; and (c) building modern high-efficiency thermal power plants.

\section{NOMENCLATURE}

\begin{tabular}{|c|c|}
\hline$P$ & pressure, $\mathrm{Pa}$ \\
\hline$s$ & specific entropy, J/kg K \\
\hline$T$ & temperature, ${ }^{\circ} \mathrm{C}$ \\
\hline$x$ & steam quality \\
\hline \multicolumn{2}{|c|}{ Subscripts } \\
\hline c & condenser \\
\hline $\mathrm{cr}$ & critical \\
\hline el & electrical \\
\hline fw & feedwater \\
\hline in & inlet \\
\hline th & thermal \\
\hline \multicolumn{2}{|c|}{ Acronyms } \\
\hline AGR & Advanced Gas-cooled Reactor \\
\hline BWR & Boiling Water Reactor \\
\hline DE-TOP & $\begin{array}{l}\text { DEesalination Thermodynamic } \\
\text { Optimization Program }\end{array}$ \\
\hline EEC & Electrical-Energy Consumption \\
\hline
\end{tabular}

$\begin{array}{ll}\text { ext. } & \text { extended } \\ \text { HDI } & \text { Human Development Index } \\ \text { HPH } & \text { High Pressure Heater } \\ \text { IAEA } & \text { International Atomic Energy Agency } \\ \text { IPT } & \text { Intermediate Pressure Turbine } \\ \text { LGR } & \text { Light-water Graphite-moderated Reactor } \\ \text { LMFBR } & \text { Liquid Metal Fast Breeder Reactor } \\ \text { LNG } & \text { Liquefied Natural Gas } \\ \text { LPH } & \text { Low Pressure Heater } \\ \text { LPT } & \text { Low Pressure Turbine } \\ \text { NPP } & \text { Nuclear Power Plant } \\ \text { PHWR } & \text { Pressurized Heavy Water Reactor } \\ \text { PP } & \text { Power Plant } \\ \text { PWR } & \text { Pressurized Water Reactor } \\ \text { VVER } & \text { Water-cooled Water-moderated Power } \\ & \text { Reactor (in Russian abbreviations) }\end{array}$

EEC Electrical-Energy Consumption 


\title{
Производство электроэнергии в мире и Украине: состояние на сегодня и развитие в будущем
}

\author{
А. Зворыкин, И. Пиоро, Н. Фиалко
}

Анотация. Производство электроэнергии является ключевым фактором развития промышленности, сельского хозяйства, технологий и уровня жизни. Также, развитая и мощьная энергетика с различными источниками энергии очень важна для независимости страны. В иелом, электричество может быть получено из: 1) не возобновляемых источников энергии, таких как уголь; природный газ; нефть; и атомная энергия; и 2) возобновляемых источников энергии, таких как гидроэнергетика; биомасса; ветряная, геотермальная, и солнечная энергии; и энергия приливов и волн. Однако основными источниками для производства электроэнергии в мире являются: 1) тепловая энергия - преимущественно уголь ( 40\%) и природный газ ( 23\%); 2) «мощчные» гидроэлектростанщии ( 17\%); и 3) ядерная энергия ( 11\%). Для остального производства электроэнергии используется нефть ( 4\%) и возобновляемые источники, такие как биомасса, ветер, геотермальные и солнечные станции ( 5\%), которые используются в отдельных странах. Кроме того, источники энергии, такие как ветер и солнце, и некоторые другие (приливные и волновые станции), являются ненадёжными поставщиками электроэнергии из-за зависимости от Матери-природы. И не могут использоваться отдельно для промьшленного производства электроэнергии.

Ядерная энергетика в Украине является важнейшим источником производства электроэнергии в стране. В настоящее время украинские Атомные ЭлектроСтаничи (АЭС) производят около 45,5\% всей электроэнергии, за которыми следуют тепловые станции ( 47,6\%) (угольные - 38\% и газовые - 9,6\%), а остальная часть - возобновляемые источники, в основном гидроэлектростаниии - 5,9\%. Атомная энергетика базируется на четырех АЭС (15 реакторов с водой под давлением (PWR), включая крупнейшую в Европе - Запорожскую АЭС с общей установленной мощностью около 6 $000 \mathrm{MBm})$.

Два из этих 15 реакторов были построены и введены в эксплуатачию в 70-е годы, десять в 80-е, один в 90-е годы и только два в 2004 году. Поэтому на основе анализа мировых энергетических реакторов с точки зрения их максимальной годовой эксплуатации (в настоящее время самые старые реакторы возрастом в 47 лет) было сделано несколько прогнозов для будущего атомной энергетики в мире и в Украине. К сожалению, все эти прогнозы весьма пессимистичны. Существует вероятность того, что около 2030-2040 г2. подавляющее большинство мировых реакторов и украинских реакторов будут закрыты, и, в частности, Украина может остаться без основного и жизненно важного источника выработки электроэнергии.

Ключевые слова: производство электроэнергии, атомная электростанция, ядерный реактор, тепловая эффективность, коэффициент мощности

\section{Виробництво електроенергії у світі та Україні: стан на сьогодні і розвиток в майбутньому}

\author{
О. Зворикін, І. Піоро, Н. Фіалко
}

Анотація. Виробництво електроенергї $є$ ключовим фактором розвитку промисловості, сільського господарства, технологій і рівня життя. Також, розвинена і потужна енергетика з різними джерелами енергї дуже важлива для незалежності краӥни. В иілому, електрику може бути отримано з: 1) невідновлюваних джерел енергії, таких як вугілля; природний газ; нафту; і атомна енергія; i 2) поновлюваних джерел енергії, таких як гідроенергетика; біомаса; вітряна, геотермальна, і сонячна енергї; і енергія припливів і хвиль. Однак основними джерелами для виробництва електроенергії в світі є: 1) теплова енергія - переважно вугілля ( 40\%) і природний газ ( 23\%); 2) «потужні» гідроелектростаниії ( 17\%); $i$ 3) ядерна енергія ( 11\%). Для решти виробництва електроенергї використовується нафту ( 4\%) $i$ поновлювані джерела, такі як біомаса, вітер, геотермальні і сонячні станції ( 5\%), які використовуються в окремих країнах. Крім того, джерела енергії, такі як вітер і соние, і деякі інші (приливні і хвильові станції), є ненадійними постачальниками електроенергії через залежність від Матері-природи. I не можуть використовуватися окремо для промислового виробниитва електроенергії.

Ядерна енергетика в Україні є найважливішим джерелом виробництва електроенергії в краӥні. В даний час українські атомні електростанції (АЕС) виробляють близько 45,5\% всісї електроенергії, за якими слідують теплові станціі ( 47,6\%) (вугільні 38\% і газові 9,6\%), а інша частина - поновлювані джерела, в основному гідроелектростанції 5,9\%. Атомна енергетика базується на чотирьох АEC (15 реакторів з водою під тиском (PWR), включаючи найбільшу в Свропі Запорізьку АЕС із загальною встановленою потужністю близько 6000 MBт).

Два з иих 15 реакторів були побудовані і введені в експлуатацію в 70-і роки, десять в 80-е, один в 90-і роки і тільки два в 2004 рочі. Тому на основі аналізу світових енергетичних реакторів з точки зору їх максимальної річної експлуатації (в даний час найстаріші реактори віком в 47 років) було зроблено кілька прогнозів для майбутнього атомної енергетики 6 світі та в Україні. На жаль, всі иі прогнози досить песимістичні. Існує ймовірність того, що близько 2030-2040 рр. переважна більшість світових реакторів і украӥнських реакторів будуть закриті, $i$, зокрема, Україна може залишитися без основного і життєво важливого джерела вироблення електроенергії.

Ключові слова: виробництво електроенергії, атомна електростанція, ядерний реактор, теплова ефективність, коефіцієнт потужності 


\section{References}

1. Dragunov, A., Saltanov, Eu., Pioro, I., Kirillov, P. and Duffey, R. (2015), Power Cycles of Generation III and III+ Nuclear Power Plants, ASME Journal of Nuclear Engineering and Radiation Science, Vol. 1, No. 2, 10 pages.

2. Grigor'ev, V.A. and Zorin, V.M. (ed.) (1988), Thermal and Nuclear Power Plants. Handbook, (In Russian), $2^{\text {nd }}$ edition, Energoatomizdat Publishing House, Moscow, Russia, 625 pages.

3. Pioro, I.L. (ed.) (2016), Handbook of Generation IV Nuclear Reactors, Woodhead Publishing (WP), Elsevier, Duxford, UK, 940 pages.

4. Human Development Report, (2013), UN Development Programme, March $14^{\text {th }}, 216$ pages.

5. IAEA (International Atomic Energy Agency), (2013), "Desalination Thermodynamic Optimization Program (DE-TOP)", available at: https://www.iaea.org/NuclearPower/Desalination/.

6. Nuclear News, (2017), March, Publication of American Nuclear Society (ANS), pp. 34-65.

7. Nuclear News, (2011), March, Publication of American Nuclear Society (ANS), pp. 45-78.

8. Pioro, I. and Duffey, R. (2015), Nuclear Power as a Basis for Future Electricity Generation, ASME Journal of Nuclear Engineering and Radiation Science, Vol. 1, No. 1, 19 pages. Free download from: http://nuclearengineering. asmedigitalcollection.asme.org/article.aspx? articleID $=2085849$.

9. Pioro, I. and Kirillov, P. (2013a), Current Status of Electricity Generation at Thermal Power Plants, Materials and Processes for Energy: Communicating Current Research and Technological Developments, Energy Book Series \#1, in Méndez-Vilas, A. (ed.), Formatex Research Center, Spain, pp. 796-805. Free download from: http://www.formatex.info/energymaterialsbook/book/796-805.pdf.

10. Pioro, I. and Kirillov, P. (2013b), Current Status of Electricity Generation at Thermal Power Plants, Materials and Processes for Energy: Communicating Current Research and Technological Developments, Energy Book Series \#1, in Méndez-Vilas, A. (ed.), Formatex Research Center, Spain, pp. 796-805. Free download from: http://www.formatex.info/energymaterialsbook/book/796-805.pdf.

11. Pioro, I. and Kirillov, P. (2013c), Current Status of Electricity Generation at Nuclear Power Plants, Materials and Processes for Energy: Communicating Current Research and Technological Developments, Energy Book Series \#1, in Méndez-Vilas, A. (ed.), Formatex Research Center, Spain, pp. 806-817. Free download from: http://www.formatex.info/energymaterialsbook/book/806-817.pdf.

12. Pioro, I. and Kirillov, P. (2013d), Generation IV Nuclear Reactors as a Basis for Future Electricity Production in the World, Materials and Processes for Energy: Communicating Current Research and Technological Developments, Energy Book Series \#1, in Méndez-Vilas, A. (ed.), Formatex Research Center, Spain, pp. 818-830. Free download from: http://www.formatex.info/energymaterialsbook/book/818-830.pdf.

13. The World Fact Book, (2013), CIA, USA, available at: https:/www.cia.gov/library/publications/the-worldfactbook/geos/ca.html. 